Fusion Energy Division

\title{
One Dimensional Global and Local Solution for ICRF
}

\section{Heating}

C. Y. Wang

D. B. Batchelor

E. F. Jaeger

M. D. Carter

DATE PUBLISHED — February 1995

Prepared for the

Office of Fusion Energy

Budget Activity AT 0520210

Prepared by

OAK RIDGE NATIONAL LABORATORY

Oak Ridge, Tennessee 37831-6285 managed by

MARTIN MARIETTA ENERGY SYSTEMS, INC.

for the

U.S. DEPARTMENT OF ENERGY

under contract DE-AC05-84OR21400

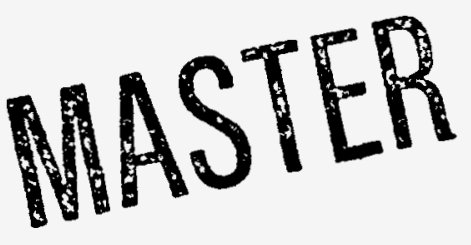




\section{DISCLAIMER}

This report was prepared as an account of work sponsored by an agency of the United States Government. Neither the United States Government nor any agency thereof, nor any of their employees, make any warranty, express or implied, or assumes any legal liability or responsibility for the accuracy, completeness, or usefulness of any information, apparatus, product, or process disclosed, or represents that its use would not infringe privately owned rights. Reference herein to any specific commercial product, process, or service by trade name, trademark, manufacturer, or otherwise does not necessarily constitute or imply its endorsement, recommendation, or favoring by the United States Government or any agency thereof. The views and opinions of authors expressed herein do not necessarily state or reflect those of the United States Government or any agency thereof. 


\section{DISCLAIMER}

Portions of this document may be illegible in electronic image products. Images are produced from the best available original document. 


\section{CONTENTS}

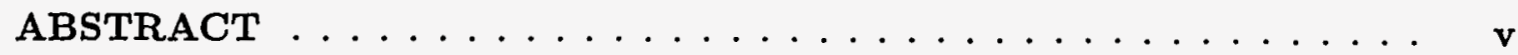

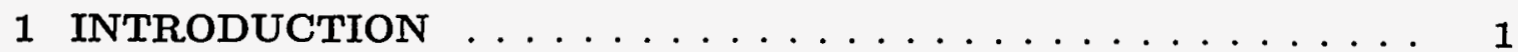

2 WAVE EQUATION ....................... 2

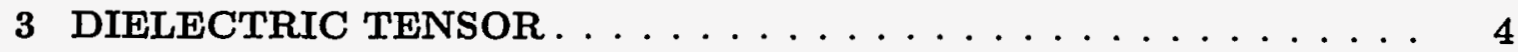

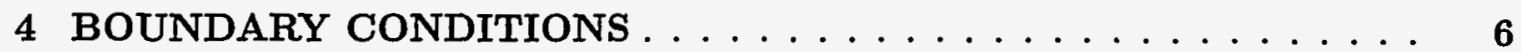

4.1 Scattering Boundary Conditions $\ldots \ldots \ldots \ldots \ldots \ldots$

4.2 Antenna in Vacuum . . . . . . . . . . . . . . . . 8

4.3 Fixed Tangential B Boundary Conditions . . . . . . . . . . . . 11

5 POWER ABSORPTION AND KINETIC ENERGY FLUX . . . . . . . 12

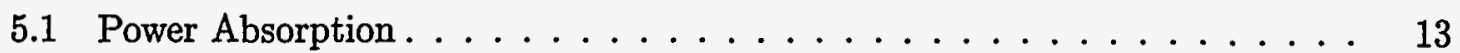

5.2 Kinetic Energy Flux . . . . . . . . . . . . . . . . 14

6 NUMERICAL METHOD . . . . . . . . . . . . . . . . 15

7 NUMERICAL RESULTS . . . . . . . . . . . . . . . 15

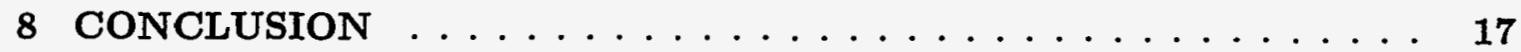

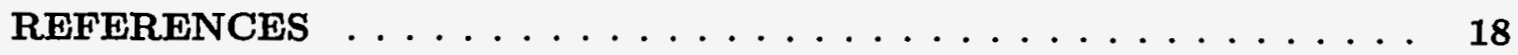





\begin{abstract}
A numerical code GLOSI [Global and Local One-dimensional Solution for Ion cyclotron range of frequencies (ICRF) heating] is developed to solve one-dimensional wave equations resulting from the use of radio frequency $(\mathrm{RF})$ waves to heat plasmas. The code uses a finite difference method. Due to its numerical stability, the code can be used to find both global and local solutions when imposed with appropriate boundary conditions. Three types of boundary conditions are introduced to describe wave scattering, antenna wave excitation, and fixed tangential wave magnetic field. The scattering boundary conditions are especially useful for local solutions. The antenna wave excitation boundary conditions can be used to excite fast and slow waves in a plasma. The tangential magnetic field boundary conditions are used to calculate impedance matrices, which describe plasma and antenna coupling and can be used by an antenna code to calculate antenna loading. These three types of boundary conditions can also be combined to describe various physical situations in RF plasma heating. The code also includes plasma thermal effects and calculates collisionless power absorption and kinetic energy flux. The plasma current density is approximated by a second-order Larmor radius expansion, which results in a sixth-order ordinary differential equation.
\end{abstract}





\section{INTRODUCTION}

The experimental success in heating plasmas on the Tokamak Fusion Test Reactor (TFTR)[1] and the Joint European Torus (JET)[2] with waves in the ion cyclotron range of frequencies (ICRF) has stimulated interest in theoretical models for calculating global and local wave fields and power deposition profiles for tokamaks. There are many onedimensional (1-D) numerical codes[3-9] which employ various numerical algorithms to find either the global or local solutions. The global solutions are usually solved in a region spanning from a conducting wall to another conducting wall with an antenna and a plasma in between, whereas the local solutions are often solved in a thin layer covering a resonance or a mode conversion to obtain the so-called scattering matrix. In application, we often find that a numerical code for any of these types of regions with very flexible boundary conditions is badly needed. For instance, in studying fast wave poloidal flow generation in an edge plasma, a code is needed to solve the sixth-order ordinary differential equations (ODEs) locally in a low plasma density region[10]. In studying plasma loading for a wave launching antenna, a code is needed to calculate plasma impedance matrices[11]. In studying electron heating and current drive by mode converted slow waves, a code is required to solve the ODEs in a region covering a mode conversion layer and a cutoff away from the mode conversion layer[12]. Thus, it is desirable to have a code which enables us to find a solution within any interval and allows various physical boundary conditions. Recently, we developed a 1-D numerical code GLOSI (Global and Local One-dimensional Solution for ICRF heating), which, due to its numerical stability, can find both global and local solutions when appropriate boundary conditions are imposed. The code uses a finite difference method to solve 1-D wave equations resulting from the use of radio frequency (RF) waves to heat plasmas. The plasma current density is approximated by a second-order Larmor radius expansion, which results in a sixth-order ODE.

The local solutions in many codes are found from a fourth-order ODE obtained from the sixth-order ODE by assuming that the parallel electric field $E_{z}$ is small and can be neglected or treated as a perturbation $[3,4,6,13]$. This is a good approximation in the region with high plasma density because there are always enough electrons to short out the parallel electric field $E_{z}$. However, GLOSI is developed to find solutions in any region, even at the plasma edge, where the electron density is low and the parallel electric field may not be small enough to be neglected or treated as a perturbation. GLOSI always solves the sixth-order ODE and allows the presence of a large parallel electric field $E_{z}$.

Three different types of boundary conditions can be used in any combination to describe various physical situations in RF plasma heating. These boundary conditions describe wave scattering, antenna wave excitation, and fixed tangential wave magnetic field. The scattering boundary conditions are especially useful for local solutions. The antenna wave excitation boundary conditions can be used to excite fast and slow waves in a plasma. The fixed tangential magnetic field boundary conditions are used to calculate impedance matrices, which describe plasma and antenna coupling and can be used by an antenna code to calculate antenna loading[14]. The code also includes plasma thermal effects and calculates collisionless power absorption and kinetic energy flux.

After appropriate boundary conditions are found for a physical situation, the finite difference method is used to solve the ODEs. This approach is fully implicit and requires the inversion of the complete finite difference matrix in one step, including boundary conditions. 
Such an implicit method does not suffer from a tendency of the evanescent wave branches to grow exponentially[7, 15]. This method was successfully tested in solving the ODEs globally[7], and its accuracy in solving a tunneling equation locally was detailed in Ref. [15]. The implementation of this method is very straightforward because there are standard twopoint boundary value ODE solvers available.

The organization of this report is as follows. In Sec. 2 we derive the wave equation solved by the code. In Sec. 3 we list dielectric tensors for the plasma current density. Section 4 presents the three types of boundary conditions for local and global solutions, and Sec. 5 presents the power absorption and kinetic energy flux used in the code. In Sec. 6 we briefly describe the numerical method used in the code. Section 7 gives several examples computed by the code, and Sec. 8 concludes with a brief summary.

\section{WAVE EQUATION}

The code considers a perpendicularly stratified, 1-D slab plasma model of a tokamak in which the equilibrium quantities are functions of the radial coordinate $x$ only and the external static magnetic field $\mathbf{B}_{0}$ is along the toroidal direction $z$. The wave electric and magnetic fields $\mathbf{E}$ and $\mathbf{B}$ are assumed to be small and with harmonic dependences of the form, $f(x) \exp \left[i\left(k_{y} y+k_{z} z-\omega t\right)\right]$, where $\omega / 2 \pi$ is the wave frequency, and $k_{y}$ and $k_{z}$ are the poloidal and toroidal wave numbers. The code solves the wave equation,

$$
-\nabla \times \nabla \times \mathbf{E}+\frac{\omega^{2}}{c^{2}} \mathbf{E}+i \omega \mu_{0} \sum_{\alpha} \mathrm{J}_{\alpha}=-i \omega \mu_{0} \mathrm{~J}_{\mathrm{ext}},
$$

where $c$ is the speed of light, $\mu_{0}$ is the vacuum permeability, $\mathbf{J}_{\text {ext }}$ represents the antenna current, and $\mathbf{J}_{\alpha}$ is the plasma current for species $\alpha$ and approximated by a finite Larmor radius expansion to the second order[7]:

$$
\begin{aligned}
\mathbf{J}_{\alpha}= & \left(-i \epsilon_{0} \omega\right)\left\{\left(\chi_{\alpha}^{(0)}+\rho_{\alpha}^{(1)}+\tau_{\alpha}^{(2)}\right) \cdot \mathbf{E}+\left[\chi_{\alpha}^{(1)}+\rho_{\alpha}^{(2)}\right]_{U} \cdot \frac{\partial \mathbf{E}}{\partial x}\right. \\
& \left.+\frac{\partial}{\partial x}\left(\left[\chi_{\alpha}^{(1)}+\rho_{\alpha}^{(2)}\right]_{L} \cdot \mathbf{E}\right)+\frac{\partial}{\partial x}\left(\chi_{\alpha}^{(2)} \cdot \frac{\partial \mathbf{E}}{\partial x}\right)\right\} .
\end{aligned}
$$

All the susceptibility tensors in Eq. (2) are listed in the next section. With $\mathbf{J}_{\alpha}$ from Eq. (2), the wave equation (1) becomes

$$
\begin{aligned}
-\frac{1}{k_{0}^{2}} \nabla \times \nabla \times \mathbf{E}+ & \left\{\left(\epsilon^{(0)}+\mu^{(1)}+\nu^{(2)}\right) \cdot \mathbf{E}+\left[\epsilon^{(1)}+\mu^{(2)}\right]_{U} \cdot \frac{\partial \mathbf{E}}{\partial x}\right. \\
+ & \left.\frac{\partial}{\partial x}\left(\left[\epsilon^{(1)}+\mu^{(2)}\right]_{L} \cdot \mathbf{E}\right)+\frac{\partial}{\partial x}\left(\epsilon^{(2)} \cdot \frac{\partial \mathbf{E}}{\partial x}\right)\right\}=-\frac{i}{\omega \epsilon_{0}} \mathbf{J}_{\text {ext }}
\end{aligned}
$$

with $k_{0}=\omega / c$,

$$
\begin{aligned}
\epsilon^{(0)} & =\mathcal{I}+\sum_{\alpha} \chi_{\alpha}^{(0)}, \\
\epsilon^{(1,2)} & =\sum_{\alpha} \chi_{\alpha}^{(1,2)}, \\
\mu^{(1,2)} & =\sum_{\alpha} \rho_{\alpha}^{(1,2)}, \\
\nu^{(2)} & =\sum_{\alpha} \tau_{\alpha}^{(2)},
\end{aligned}
$$


where $\mathcal{I}$ is the $3 \times 3$ unit matrix. In $(x, y, z)$ coordinates, we can write

$$
-\frac{1}{k_{0}^{2}} \nabla \times \nabla \times \mathbf{E}=\left(\mathcal{A}_{0} \frac{\partial^{2}}{\partial x^{2}}+\mathcal{B}_{0} \frac{\partial}{\partial x}+\mathcal{C}_{0}\right) \cdot \mathbf{E}
$$

where

$$
\begin{gathered}
\mathcal{A}_{0}=\frac{1}{k_{0}^{2}}\left[\begin{array}{lll}
0 & 0 & 0 \\
0 & 1 & 0 \\
0 & 0 & 1
\end{array}\right], \\
\mathcal{B}_{0}=\frac{1}{k_{0}^{2}}\left[\begin{array}{ccc}
0 & -i k_{y} & -i k_{z} \\
-i k_{y} & 0 & 0 \\
-i k_{z} & 0 & 0
\end{array}\right],
\end{gathered}
$$

and

$$
\mathcal{C}_{0}=\frac{1}{k_{0}^{2}}\left[\begin{array}{ccc}
-k_{y}^{2}-k_{z}^{2} & 0 & 0 \\
0 & -k_{z}^{2} & k_{y} k_{z} \\
0 & k_{z} k_{y} & -k_{y}^{2}
\end{array}\right]
$$

By using Eq. (8) we can rewrite the wave equation [Eq. (3)] as

$$
\frac{\partial}{\partial x}\left(\mathcal{P} \cdot \frac{\partial \mathbf{E}}{\partial x}+\mathcal{Q} \cdot \mathbf{E}\right)+\mathcal{R} \cdot \frac{\partial \mathbf{E}}{\partial x}+\mathcal{S} \cdot \mathbf{E}=\frac{-i}{\epsilon_{0} \omega} \mathrm{J}_{\text {ext }},
$$

with

$$
\begin{aligned}
\mathcal{P} & =\mathcal{A}_{0}+\epsilon^{(2)}, \\
\mathcal{Q} & =\left[\epsilon^{(1)}+\mu^{(2)}\right]_{L}, \\
\mathcal{R} & =\mathcal{B}_{0}+\left[\epsilon^{(1)}+\mu^{(2)}\right]_{U}, \\
\mathcal{S} & =\mathcal{C}_{0}+\epsilon^{(0)}+\mu^{(1)}+\nu^{(2)}
\end{aligned}
$$

Equation (12) is a set of three second-order ODEs. To convert Eq. (12) to a set of six first-order ODEs, we first define

$$
\mathbf{F}=\mathcal{P} \cdot \frac{\partial \mathbf{E}}{\partial x}+\mathcal{Q} \cdot \mathbf{E}
$$

and then Eq. (12) becomes

$$
\frac{\partial}{\partial x}\left[\begin{array}{l}
\mathbf{E} \\
\mathbf{F}
\end{array}\right]=\left[\begin{array}{cc}
-\mathcal{P}^{-1} \cdot \mathcal{Q} & \mathcal{P}^{-1} \\
\mathcal{R} \cdot \mathcal{P}^{-1} \cdot \mathcal{Q}-\mathcal{S} & -\mathcal{R} \cdot \mathcal{P}^{-1}
\end{array}\right]\left[\begin{array}{l}
\mathbf{E} \\
\mathbf{F}
\end{array}\right]+\left[\begin{array}{c}
0 \\
\left(-i / \epsilon_{0} \omega\right) \mathbf{J}_{\text {ext }}
\end{array}\right]
$$

where the superscript "-1" designates the matrix inversion. Equation (18) is a set of six first-order ODEs and requires six boundary conditions to become a well-posed problem. The various boundary conditions are detailed in Sec. 4 . 


\section{DIELECTRIC TENSOR}

We approximate the plasma current $\mathrm{J}_{\alpha}$ for each species $\alpha$ by a finite Larmor radius expansion to the second order, Eq. (2), where (dropping subscript $\alpha$ )

$$
\begin{gathered}
\chi^{(0)}=\left[\begin{array}{ccc}
\chi_{x x}^{(0)} & \chi_{x y}^{(0)} & 0 \\
\chi_{y x}^{(0)} & \chi_{y y}^{(0)} & 0 \\
0 & 0 & \chi_{z z}^{(0)}
\end{array}\right], \\
\rho^{(1)}=\left[\begin{array}{ccc}
0 & 0 & \rho_{x z}^{(1)} \\
0 & 0 & \rho_{y z}^{(1)} \\
\rho_{z x}^{(1)} & \rho_{z y}^{(1)} & 0
\end{array}\right], \\
\tau^{(2)}=\left[\begin{array}{ccc}
\tau_{x x}^{(2)} & \tau_{x y}^{(2)} & 0 \\
\tau_{y x}^{(2)} & \tau_{y y}^{(2)} & 0 \\
0 & 0 & \tau_{z z}^{(2)}
\end{array}\right], \\
{\left[\chi^{(1)}+\rho^{(2)}\right]_{U}=\left[\begin{array}{ccc}
0 & \rho_{x y}^{(2)} & \chi_{x z}^{(1)} \\
0 & 0 & \chi_{y z}^{(1)} \\
0 & 0 & 0
\end{array}\right],} \\
{\left[\chi^{(1)}+\rho^{(2)}\right]_{L}=\left[\begin{array}{ccc}
0 & 0 & 0 \\
\rho_{y x}^{(2)} & 0 & 0 \\
\chi_{z x}^{(1)} & \chi_{z y}^{(1)} & 0
\end{array}\right],}
\end{gathered}
$$

and

$$
\chi^{(2)}=\left[\begin{array}{ccc}
\chi_{x x}^{(2)} & \chi_{x y}^{(2)} & 0 \\
\chi_{y x}^{(2)} & \chi_{y y}^{(2)} & 0 \\
0 & 0 & \chi_{z z}^{(2)}
\end{array}\right]
$$

The nonzero elements of the tensors listed below come from Jaeger, Batchelor, and Weitzner[7], but several gradient terms are neglected. The justification for neglecting these terms was discussed by Brambilla[16]. We first introduce the notation

$$
\begin{aligned}
& \ell=\frac{\omega_{p}^{2}}{\omega^{2}} \xi_{0} Z_{1} \\
& r=\frac{\omega_{p}^{2}}{\omega^{2}} \xi_{0} Z_{-1} \\
& p=-\frac{\omega_{p}^{2}}{\omega^{2}} \xi_{0}^{2} Z_{0}^{\prime}
\end{aligned}
$$

and $[16]$ 


$$
\begin{aligned}
\lambda_{n} & =\frac{1}{2} \frac{\omega_{p}^{2}}{\Omega^{2}} \frac{v_{t h}^{2}}{c^{2}}\left(-\xi_{0} Z_{n}\right) \\
\pi_{n} & =\frac{1}{2} \frac{\omega_{p}^{2}}{\Omega^{2}} \frac{v_{t h}^{2}}{c^{2}} \xi_{0} \xi_{n} Z_{n}^{\prime} \\
\zeta_{n} & =\frac{1}{2} \frac{\omega_{p}^{2}}{\omega \Omega} \frac{v_{t h}^{2}}{c^{2}} \xi_{0}^{2} Z_{n}^{\prime},
\end{aligned}
$$

where $\xi_{n}=(\omega-n \Omega) / k_{z} v_{t h}, Z_{n}=Z\left(\xi_{n}\right)$ is the plasma dispersion function[17], and $Z_{n}^{\prime}=$ $d Z\left(\xi_{n}\right) / d \xi_{n}$. Here, $\Omega$ is the cyclotron frequency with its sign of the charge, $\omega_{p}$ is the plasma frequency, and $v_{t h}$ is the thermal speed.

We also define

$$
\begin{aligned}
s & =(r+\ell) / 2 \\
d & =(r-\ell) / 2 \\
\sigma_{n} & =\left(\lambda_{-n}+\lambda_{n}\right) / 2 \\
\delta_{n} & =\left(\lambda_{-n}-\lambda_{n}\right) / 2
\end{aligned}
$$

Note that $\ell, r, p, s$, and $d$ are related to the widely used Stix notation[18] for a cold plasma by

$$
\begin{aligned}
& L=1+\sum_{\alpha} \ell_{\alpha} \\
& R=1+\sum_{\alpha} r_{\alpha}, \\
& P=1+\sum_{\alpha} p_{\alpha} \\
& S=1+\sum_{\alpha} s_{\alpha} \\
& D=\sum_{\alpha} d_{\alpha}
\end{aligned}
$$

as plasma temperature $T_{\alpha} \rightarrow 0$.

By using the above notation, the nonzero elements of the tensors can be expressed as

$$
\begin{aligned}
& \chi_{x x}^{(0)}=\chi_{y y}^{(0)}=s, \\
& \chi_{x y}^{(0)}=-\chi_{y x}^{(0)}=-i d, \\
& \chi_{z z}^{(0)}=p, \\
& \chi_{x z}^{(1)}=\chi_{z x}^{(1)}=\left(-\frac{i k_{z}}{2 k_{0}^{2}}\right)\left(\zeta_{-1}-\zeta_{1}\right), \\
& \chi_{y z}^{(1)}=-\chi_{z y}^{(1)}=\left(-\frac{k_{z}}{2 k_{0}^{2}}\right)\left(2 \zeta_{0}-\zeta_{-1}-\zeta_{1}\right), \\
& \chi_{z x}^{(2)}=\left(\frac{1}{k_{0}^{2}}\right)\left(\sigma_{2}-\sigma_{1}\right),
\end{aligned}
$$




$$
\begin{gathered}
\chi_{y y}^{(2)}=\left(\frac{1}{k_{0}^{2}}\right)\left(\sigma_{2}-3 \sigma_{1}+2 \lambda_{0}\right), \\
\chi_{x y}^{(2)}=-\chi_{y x}^{(2)}=\left(-\frac{i}{k_{0}^{2}}\right)\left(\delta_{2}-2 \delta_{1}\right), \\
\chi_{z z}^{(2)}=\left(\frac{1}{2 k_{0}^{2}}\right)\left(\pi_{-1}+\pi_{1}-2 \pi_{0}\right), \\
\rho_{x z}^{(1)}=-\rho_{z x}^{(1)}=-i k_{y} \chi_{y z}^{(1)}, \\
\rho_{y z}^{(1)}=\rho_{z y}^{(1)}=\left(-\frac{k_{z}}{k_{0}^{2}}\right) \frac{d \zeta_{0}}{d x}+i k_{y} \chi_{x z}^{(1)}, \\
\rho_{x y}^{(2)}=\rho_{y x}^{(2)}=-\left(\frac{i}{k_{0}^{2}}\right) \frac{d \delta_{1}}{d x}+\left(\frac{2 i k_{y}}{k_{0}^{2}}\right)\left(\sigma_{1}-\lambda_{0}\right), \\
\tau_{x y}^{(2)}=\left(\frac{1}{2 k_{0}^{2}}\right)\left[-\frac{d^{2} \sigma_{1}}{d x^{2}}-2 k_{y} \frac{d}{d x}\left(\delta_{1}-\delta_{2}\right)+2 k_{y} \frac{\Omega}{\omega} \frac{d \sigma_{1}}{d x}\right]-k_{y}^{2} \chi_{y y}^{(2)}, \\
\tau_{z z}^{(2)}=\left(\frac{i}{2 k_{0}^{2}}\right)\left[\frac{d^{2} \delta_{1}}{d x^{2}}+2 k_{y} \frac{d}{d x}\left(\sigma_{1}-\sigma_{2}\right)-2 k_{y} \frac{\Omega}{\omega} \frac{d \delta_{1}}{d x}\right]-k_{y}^{2} \chi_{x y}^{(2)}, \\
\tau_{y y}^{(2)}=\left(\frac{1}{2 k_{0}^{2}}\right)\left[-3 \frac{d^{2} \sigma_{1}}{d x^{2}}+2 k_{y} \frac{d}{d x}\left(\delta_{1}+\delta_{2}\right)+2 k_{y} \frac{\Omega}{\omega} \frac{d \sigma_{1}}{d x}\right]-k_{y}^{2} \chi_{x x}^{(2)}, \\
\left.=\frac{d^{2} \pi_{0}}{d x^{2}}-k_{y} \frac{d}{d x}\left(\pi_{-1}-\pi_{1}\right)+2 k_{y} \frac{\Omega}{\omega} \frac{d \pi_{0}}{d x}\right]-k_{y}^{2} \chi_{z z}^{(2)} .
\end{gathered}
$$

This set of susceptibility tensors is equivalent to that in Ref. [16] except for a slight difference in the drift current [i.e., terms in $\tau^{(2)}$ containing a factor of $k_{y}(\Omega / \omega)$ ].

\section{BOUNDARY CONDITIONS}

The code allows three different types of boundary conditions at each boundary. The first type is the scattering boundary condition that specifies which waves go out and which waves come in. This boundary condition is especially useful for local solutions. The second type is the plasma wave excitation from an antenna in vacuum. The solutions for the wave equation in vacuum can be solved analytically. The analytical vacuum solutions can be used to construct the boundary conditions at the plasma and vacuum interface. The third type is to impose a fixed tangential magnetic field in the vacuum, and is used to calculate the impedance matrix for antenna and plasma coupling.

Equation (18) is a set of six first-order ODEs and requires six boundary conditions. Since the ODEs are homogenous, the amplitudes of waves are determined up to a normalization. In the case of antenna wave excitation, the normalization can be the power emitted by the antenna. In the cases of scattering boundary conditions, the normalization of $E_{y}=1$ at one boundary can be used. In the following subsections, we discuss the three types of boundary conditions in detail. 


\subsection{Scattering Boundary Conditions}

There are many possible scattering boundary conditions. For example, if we have a fast wave incident from the low field side, then on the low field side there are incident and reflected fast waves and outgoing (in terms of total energy flux defined in Sec. 5) ion Bernstein and shear Alfvén waves. On the high field side, there are a transmitted fast wave and outgoing ion Bernstein and shear Alfvén waves. A few other variants include a fast wave incident from the high field side, or an ion Bernstein wave (IBW) incident from the low field side or high field side. It is even possible to have a set of scattering boundary conditions to describe the transmitted fast wave reflected from a perfectly conducting wall[19]. In our code, we can also impose a set of scattering boundary conditions at one boundary and the antenna wave excitation or the tangential magnetic field boundary conditions at the other boundary. Rather than try to list all variants of the scattering conditions, we describe the scattering boundary conditions for a fast wave incident from the low field side in detail. Other variants can be obtained in the same fashion without any difficulty.

The asymptotic solution for $x$ away from a resonance can be expanded in terms of Wentzel-Kramers-Brillouin (WKB) modes. We assume that the WKB mode is

$$
\mathbf{y} \equiv\left[\begin{array}{l}
\mathbf{E} \\
\mathbf{F}
\end{array}\right]=\mathbf{Y} \exp \left[i \int^{x} k\left(x^{\prime}\right) d x^{\prime}\right],
$$

where $\mathrm{Y}$ is a vector with six elements and has weak dependence on $x$. Substituting $\mathrm{y}$ into Eq. (18), we obtain a characteristic equation,

$$
\operatorname{det}\left[\begin{array}{cc}
-\mathcal{P}^{-1} \cdot \mathcal{Q}-k \mathcal{I} & \mathcal{P}^{-1} \\
\mathcal{R} \cdot \mathcal{P}^{-1} \cdot \mathcal{Q}-\mathcal{S} & -\mathcal{R} \cdot \mathcal{P}^{-1}-k \mathcal{I}
\end{array}\right]=0
$$

The characteristic equation gives six eigenvalues, $k_{i}(i=1,2, \ldots, 6)$. Each eigenvalue $k_{i}$ has its corresponding eigenvector, $Y_{i}=\left(Y_{1 i}, Y_{2 i}, Y_{3 i}, Y_{4 i}, Y_{5 i}, Y_{6 i}\right)^{T}$, and the superscript $T$ designates the matrix transpose. We assume that $k_{1}$ and $k_{2}$ are fast waves traveling in the $-x$ and $+x$ directions, $k_{3}$ and $k_{4}$ are IBWs with energy fluxes flowing in the $-x$ and $+x$ directions, and $k_{5}$ and $k_{6}$ are shear Alfvén waves with energy fluxes flowing in the $-x$ and $+x$ directions.

On the high field side of the mode conversion layer, we expand the solution at $x=x^{-}$ as

$$
\mathrm{y}=\sum_{i=1}^{6} C_{i}^{-} \mathbf{Y}_{i}^{-},
$$

where $Y_{i}^{-}$are the eigenvectors corresponding to eigenvalues $k_{i}^{-}$, and the exponential factors $\exp \left(i \int^{x} k_{i}^{-} d x^{\prime}\right)$ are absorbed into the unknown expansion coefficients $C_{i}^{-}$. For a fast wave incident from the low field side, we have a transmitted fast wave $k_{1}^{-}$, an IBW having an outgoing energy flux $k_{3}^{-}$, and a shear Alfvén wave having an outgoing energy flux $k_{5}^{-}$on the high field side. To eliminate the other three unphysical modes $k_{2}^{-}, k_{4}^{-}$, and $k_{6}^{-}$, we simply set $C_{2}^{-}=C_{4}^{-}=C_{6}^{-}=0$ in Eq. (58). For Eq. (58) to have nontrivial $C_{1}^{-}, C_{3}^{-}$, and $C_{5}^{-}$, we obtain from Eq. (58),

$$
\operatorname{det}\left[\begin{array}{llll}
y_{1} & Y_{11}^{-} & Y_{13}^{-} & Y_{15}^{-} \\
y_{2} & Y_{21}^{-} & Y_{23}^{-} & Y_{25}^{-} \\
y_{3} & Y_{31}^{-} & Y_{33}^{-} & Y_{35}^{-} \\
y_{4} & Y_{41}^{-} & Y_{43}^{-} & Y_{45}^{-}
\end{array}\right]=0
$$




$$
\operatorname{det}\left[\begin{array}{llll}
y_{2} & Y_{21}^{-} & Y_{23}^{-} & Y_{25}^{-} \\
y_{3} & Y_{31}^{-} & Y_{33}^{-} & Y_{35}^{-} \\
y_{4} & Y_{41}^{-} & Y_{43}^{-} & Y_{45}^{-} \\
y_{5} & Y_{51}^{-} & Y_{53}^{-} & Y_{55}^{-}
\end{array}\right]=0
$$

and

$$
\operatorname{det}\left[\begin{array}{llll}
y_{3} & Y_{31}^{-} & Y_{33}^{-} & Y_{35}^{-} \\
y_{4} & Y_{41}^{-} & Y_{43}^{-} & Y_{45}^{-} \\
y_{5} & Y_{51}^{-} & Y_{53}^{-} & Y_{55}^{-} \\
y_{6} & Y_{61}^{-} & Y_{63}^{-} & Y_{65}^{-}
\end{array}\right]=0
$$

where $y_{1}, y_{2}, \cdots, y_{6}$ are the six elements of $\mathbf{y}$.

On the lower field side of the mode conversion layer, we have incident and reflected fast waves $k_{1}^{+}$and $k_{2}^{+}$, an IBW having an outgoing energy flux $k_{4}^{+}$, and a shear Alfvén wave having an outgoing energy flux $k_{6}^{+}$. We expand the solution at $x=x^{+}$as

$$
\mathrm{y}=\sum_{i=1}^{6} C_{i}^{+} \mathbf{Y}_{i}^{+}
$$

To eliminate the modes with incoming energy fluxes $k_{3}^{+}$and $k_{5}^{+}$, we set $C_{3}^{+}=C_{5}^{+}=0$ in Eq. (62). For Eq. (62) to have nontrivial $C_{1}^{+}, C_{2}^{+}, C_{4}^{+}$, and $C_{6}^{+}$, we obtain from Eq. (62),

$$
\operatorname{det}\left[\begin{array}{lllll}
y_{1} & Y_{11}^{+} & Y_{12}^{+} & Y_{14}^{+} & Y_{16}^{+} \\
y_{2} & Y_{21}^{+} & Y_{22}^{+} & Y_{24}^{+} & Y_{26}^{+} \\
y_{3} & Y_{31}^{+} & Y_{32}^{+} & Y_{34}^{+} & Y_{36}^{+} \\
y_{4} & Y_{41}^{+} & Y_{42}^{+} & Y_{44}^{+} & Y_{46}^{+} \\
y_{5} & Y_{51}^{+} & Y_{52}^{+} & Y_{54}^{+} & Y_{56}^{+}
\end{array}\right]=0
$$

and

$$
\operatorname{det}\left[\begin{array}{ccccc}
y_{2} & Y_{21}^{+} & Y_{22}^{+} & Y_{24}^{+} & Y_{26}^{+} \\
y_{3} & Y_{31}^{+} & Y_{32}^{+} & Y_{34}^{+} & Y_{36}^{+} \\
y_{4} & Y_{41}^{+} & Y_{42}^{+} & Y_{44}^{+} & Y_{46}^{+} \\
y_{5} & Y_{51}^{+} & Y_{52}^{+} & Y_{54}^{+} & Y_{56}^{+} \\
y_{6} & Y_{61}^{+} & Y_{62}^{+} & Y_{64}^{+} & Y_{66}^{+}
\end{array}\right]=0
$$

We solve the set of first-order ODEs (18) with the boundary conditions given by Eqs. (59) $-(61)$ at $x=x^{-}$and Eqs. (63) and (64) at $x=x^{+}$. A normalization $E_{y}=1$ is used at one of the two ends. This is a well-posed two-point value problem: six first-order ODEs and six boundary conditions. Here, $x^{-}$and $x^{+}$are chosen far enough from the mode conversion layer so that the asymptotic WKB expansion is valid. In the code, the WKB eigenvalues are numerically calculated from Eq. (57) by an eigenvalue solver, and each mode is numerically identified.

\subsection{Antenna in Vacuum}

Plasma waves can be excited by an antenna located outside the plasma. In this subsection we consider how to impose the boundary conditions at the plasma and vacuum interface $x=a$ with an external current source (an antenna). We assume that there is an external current source (antenna), 


$$
\mathrm{J}_{\mathrm{ext}}=\mathrm{J}_{0} \delta(x-d)
$$

outside the plasma at $x=d>a$. We assume that the antenna current has no radial component, i.e., $\mathbf{J}_{0}=J_{0 y} \hat{\mathbf{y}}+J_{0 z} \hat{\mathbf{z}}$, where $\hat{\mathbf{y}}$ and $\hat{\mathbf{z}}$ are the unit vectors along $y$ and $z$ directions, respectively. The toroidal current $J_{0 y}$ excites a fast wave, and the poloidal current $J_{0 z}$ excites an IBW in the plasma[5]. We also assume that a perfectly conducting wall is located at $x=b>d$.

With a perfectly conducting wall at $x=b$, the vacuum solution for region $x \in(d, b]$ is

$$
\begin{aligned}
& E_{y}=D_{1} \sinh [\lambda(x-b)] \\
& E_{z}=D_{3} \sinh [\lambda(x-b)], \\
& E_{x}=-\left(i / \lambda^{2}\right)\left(k_{y} d E_{y} / d x+k_{z} d E_{z} / d x\right),
\end{aligned}
$$

where $\lambda=\left(k_{y}^{2}+k_{z}^{2}-k_{0}^{2}\right)^{1 / 2}$ for $k_{y}^{2}+k_{z}^{2}-k_{0}^{2} \geq 0$ and $\lambda=i\left(k_{0}^{2}-k_{y}^{2}-k_{z}^{2}\right)^{1 / 2}$ for $k_{y}^{2}+k_{z}^{2}-k_{0}^{2}<0$. Equations (66) and (67) satisfy the perfectly conducting wall boundary condition $E_{y}(b)=$ $E_{z}(b)=0$. The vacuum solution for region $x \in(a, d)$ is

$$
\begin{aligned}
& E_{y}=C_{1} \sinh [\lambda(x-b)]+C_{2} \cosh [\lambda(x-b)], \\
& E_{z}=C_{3} \sinh [\lambda(x-b)]+C_{4} \cosh [\lambda(x-b)] .
\end{aligned}
$$

To find the connection condition at $x=d$, we integrate the three components of wave equation (1) over $x \in\left(d^{-}, d^{+}\right)$and obtain

$$
\begin{aligned}
-i k_{y}\left[E_{y}\right]-i k_{z}\left[E_{z}\right] & =0 \\
{\left[d E_{y} / d x\right]-i k_{y}\left[E_{x}\right] } & =-i \mu_{0} \omega J_{0 y} \\
{\left[d E_{z} / d x\right]-i k_{z}\left[E_{x}\right] } & =-i \mu_{0} \omega J_{0 z}
\end{aligned}
$$

where $[f(x)] \equiv f\left(d^{+}\right)-f\left(d^{-}\right)$. To connect the vacuum solutions on the two sides of the antenna, we need four connection conditions. The other one can be obtained by integrating $\nabla \cdot \mathbf{B}=0$

$$
\left[B_{x}\right]=(1 / \omega)\left(k_{y}\left[E_{z}\right]-k_{z}\left[E_{y}\right]\right)=0 .
$$

From the above four equations and Eq. (68), we obtain the connection conditions at $x=d$,

$$
\begin{aligned}
{\left[E_{y}\right] } & =\left[E_{z}\right]=0 \\
{\left[d E_{y} / d x\right] } & =\gamma_{2} \equiv i \mu_{0} \omega\left[J_{0 z}\left(k_{y} k_{z} / k_{0}^{2}\right)-J_{0 y}\left(k_{z}^{2}-\lambda^{2}\right) / k_{0}^{2}\right] \\
{\left[d E_{z} / d x\right] } & =\gamma_{3} \equiv i \mu_{0} \omega\left[J_{0 y}\left(k_{y} k_{z} / k_{0}^{2}\right)-J_{0 z}\left(k_{z}^{2}-\lambda^{2}\right) / k_{0}^{2}\right]
\end{aligned}
$$

Substituting Eqs. (66), (67), (69), and (70) into the above connection conditions, we obtain

$$
\begin{aligned}
& C_{1}=D_{1} \alpha_{2}-\gamma_{2}, \\
& C_{2}=D_{1} \alpha_{1}, \\
& C_{3}=D_{3} \alpha_{2}-\gamma_{3}, \\
& C_{4}=D_{3} \alpha_{1},
\end{aligned}
$$

with $\alpha_{1}=\sinh [\lambda(d-b)]$ and $\alpha_{2}=\cosh [\lambda(d-b)]$. 
At $x=a^{+}$, we obtain from Eqs. (69) and (70),

$$
\begin{aligned}
E_{y}\left(a^{+}\right) & =C_{1} \beta_{1}+C_{2} \beta_{2}, \\
E_{z}\left(a^{+}\right) & =C_{3} \beta_{1}+C_{4} \beta_{2}, \\
d E_{y}\left(a^{+}\right) / d x & =\lambda\left(C_{1} \beta_{2}+C_{2} \beta_{1}\right), \\
d E_{z}\left(a^{+}\right) / d x & =\lambda\left(C_{3} \beta_{2}+C_{4} \beta_{1}\right),
\end{aligned}
$$

with $\beta_{1}=\sinh [\lambda(a-d)]$ and $\beta_{2}=\cosh [\lambda(a-d)]$. Substituting $C_{1}, C_{2}, C_{3}$, and $C_{4}$ from Eqs. (78)-(81) into the above equations and further eliminating $D_{1}$ and $D_{3}$, we obtain,

$$
\begin{aligned}
& d E_{y}\left(a^{+}\right) / d x=a_{11} E_{y}\left(a^{+}\right)+a_{12} \\
& d E_{z}\left(a^{+}\right) / d x=a_{21} E_{z}\left(a^{+}\right)+a_{22}
\end{aligned}
$$

with

$$
\begin{aligned}
& a_{11}=a_{21}=\lambda\left(\alpha_{1} \beta_{1}+\alpha_{2} \beta_{2}\right) /\left(\alpha_{2} \beta_{1}+\alpha_{1} \beta_{2}\right) \\
& a_{12}=\lambda\left(\beta_{1}^{2}-\beta_{2}^{2}\right) \alpha_{1} \gamma_{2} /\left(\alpha_{2} \beta_{1}+\alpha_{1} \beta_{2}\right) \\
& a_{22}=\lambda\left(\beta_{1}^{2}-\beta_{2}^{2}\right) \alpha_{1} \gamma_{3} /\left(\alpha_{2} \beta_{1}+\alpha_{1} \beta_{2}\right)
\end{aligned}
$$

Equations (86) and (87) are for the vacuum side of the plasma and vacuum interface. To find the boundary conditions for the ODE at the plasma side of the interface $x=a^{-}$, we integrate the ODE (12) over region $x \in\left(a^{-}, a^{+}\right)$and obtain

$$
\left.(\mathbf{F}+\mathcal{R} \cdot \mathbf{E})\right|_{x=a^{-}}=\left.(\mathbf{F}+\mathcal{R} \cdot \mathbf{E})\right|_{x=a^{+}}
$$

where no presence of any external surface current is assumed at the plasma and vacuum interface. On the vacuum side $\left(x=a^{+}\right)$, we have

$$
\left.(\mathbf{F}+\mathcal{R} \cdot \mathbf{E})\right|_{x=a^{+}}=\frac{1}{k_{0}^{2}}\left[\begin{array}{c}
-i\left(k_{y} E_{y}+k_{z} E_{z}\right) \\
d E_{y} / d x-i k_{y} E_{x} \\
d E_{z} / d x-i k_{z} E_{x}
\end{array}\right]
$$

Substituting Eqs. (86) and (87) into the above equation and using $E_{y}\left(a^{-}\right)=E_{y}\left(a^{+}\right)$and $E_{z}\left(a^{-}\right)=E_{z}\left(a^{+}\right)$, we can write the boundary conditions (91) at $x=a^{-}$as

$$
\left(\mathcal{R}-\mathcal{R}_{1}\right) \cdot \mathbf{E}+\mathbf{F}=\mathbf{J}_{1}
$$

where

$$
\mathcal{R}_{1}=\frac{1}{k_{0}^{2}}\left[\begin{array}{ccc}
0 & -i k_{y} & -i k_{z} \\
0 & \left(1-k_{y}^{2} / \lambda^{2}\right) a_{11} & -\left(k_{y} k_{z} / \lambda^{2}\right) a_{21} \\
0 & -\left(k_{y} k_{z} / \lambda^{2}\right) a_{11} & \left(1-k_{z}^{2} / \lambda^{2}\right) a_{21}
\end{array}\right]
$$

and

$$
\mathbf{J}_{1}=\frac{1}{k_{0}^{2}}\left[\begin{array}{c}
0 \\
\left(1-k_{y}^{2} / \lambda^{2}\right) a_{12}-\left(k_{y} k_{z} / \lambda^{2}\right) a_{22} \\
\left(1-k_{z}^{2} / \lambda^{2}\right) a_{22}-\left(k_{y} k_{z} / \lambda^{2}\right) a_{12}
\end{array}\right]
$$


Equation (93) is imposed on the ODEs (18) at $x=a^{-}$for antenna wave excitation problems.

To see the physical meaning of Eq. (93) or Eq. (91), let us first assume that both sides of $x=a$ are vacuum. Then, the $x$-component of Eq. (91) becomes trivial if $E_{y}\left(a^{-}\right)=E_{y}\left(a^{+}\right)$ and $E_{z}\left(a^{-}\right)=E_{z}\left(a^{+}\right)$. The $y$ - and $z$-components give continuity of the tangential magnetic field: $B_{y}\left(a^{-}\right)=B_{y}\left(a^{+}\right)$and $B_{z}\left(a^{-}\right)=B_{z}\left(a^{+}\right)$. Since in vacuum Eq. (18), which is a sixth-order ODE in a plasma, becomes a fourth-order ODE, the lack of the third boundary condition does not cause any problem. If the $x<a$ side is a plasma with very low density and temperature, we find that to the leading order the $y$ - and $z$-components of Eq. (91) still lead to the continuity of the tangential magnetic field. However, the $x$-component becomes nontrivial. This additional boundary condition ensures that the problem remains well posed.

\subsection{Fixed Tangential B Boundary Conditions}

In calculating antenna loading, it is convenient to characterize wave propagation into the plasma in terms of an effective plasma surface impedance matrix relating the tangential electric and magnetic fields. The impedance matrix[14] at $x$

$$
\mathcal{Z}=\left[\begin{array}{ll}
Z_{11} & Z_{12} \\
Z_{21} & Z_{22}
\end{array}\right]
$$

is defined by

$$
\begin{aligned}
& E_{y}=\left(Z_{11} B_{y}+Z_{12} B_{z}\right) / \mu_{0} \\
& E_{z}=\left(Z_{21} B_{y}+Z_{22} B_{z}\right) / \mu_{0} .
\end{aligned}
$$

To calculate $\mathcal{Z}$, we need to solve the wave equations twice. First, we choose $B_{y}=1 \mathrm{~T}$ and $B_{z}=0$ and solve $E_{y}$ and $E_{z}$ from the wave equations and obtain $Z_{11}$ and $Z_{21}$. Then, we impose $B_{y}=0$ and $B_{z}=1 \mathrm{~T}$ and find $Z_{12}$ and $Z_{22}$.

We impose more general boundary conditions,

$$
\begin{aligned}
& B_{y}=B_{y 0}, \\
& B_{z}=B_{z 0},
\end{aligned}
$$

at $x=d>a$ in vacuum. The idea is to solve the vacuum solutions analytically first, and to use the analytical solutions to construct a set of boundary conditions of the ODEs at the plasma side of the plasma and vacuum interface. In region $x \in(a, d]$, we have the vacuum solution for the wave equation (1),

$$
\begin{aligned}
& E_{y}=(1 / \lambda) d E_{y}(d) / d x \sinh [\lambda(x-d)]+E_{y}(d) \cosh [\lambda(x-d)] \\
& E_{z}=(1 / \lambda) d E_{z}(d) / d x \sinh [\lambda(x-d)]+E_{z}(d) \cosh [\lambda(x-d)]
\end{aligned}
$$

From Faraday's law, we have

$$
\begin{aligned}
& B_{y}=-(i / \omega)\left(i k_{z} E_{x}-d E_{z} / d x\right) \\
& B_{z}=(i / \omega)\left(i k_{y} E_{x}-d E_{y} / d x\right)
\end{aligned}
$$

which, together with Eq. (68), allow us to express $d E_{y}(d) / d x$ and $d E_{z}(d) / d x$ in terms of $B_{y 0}$ and $B_{z 0}$ : 


$$
\begin{aligned}
& d E_{y}(d) / d x=\left(i \omega \lambda^{2} / k_{0}^{2}\right)\left[\left(k_{y} k_{z} / \lambda^{2}\right) B_{y 0}+\left(k_{z}^{2} / \lambda^{2}-1\right) B_{z 0}\right] \\
& d E_{z}(d) / d x=-\left(i \omega \lambda^{2} / k_{0}^{2}\right)\left[\left(k_{y} k_{z} / \lambda^{2}\right) B_{z 0}+\left(k_{y}^{2} / \lambda^{2}-1\right) B_{y 0}\right] .
\end{aligned}
$$

At $x=a^{+}$, we obtain from Eqs. (101) and (102):

$$
\begin{aligned}
E_{y}\left(a^{+}\right) & =(1 / \lambda) d E_{y}(d) / d x \beta_{1}+E_{y}(d) \beta_{2} \\
E_{z}\left(a^{+}\right) & =(1 / \lambda) d E_{z}(d) / d x \beta_{1}+E_{z}(d) \beta_{2}, \\
d E_{y}\left(a^{+}\right) / d x & =d E_{y}(d) / d x \beta_{2}+\lambda E_{y}(d) \beta_{1} \\
d E_{z}\left(a^{+}\right) / d x & =d E_{z}(d) / d x \beta_{2}+\lambda E_{z}(d) \beta_{1}
\end{aligned}
$$

with $\beta_{1}=\sinh [\lambda(a-d)]$ and $\beta_{2}=\cosh [\lambda(a-d)]$. Eliminating $E_{y}(d)$ and $E_{z}(d)$ among the above equations, we obtain the boundary conditions at $x=a^{+}$:

$$
\begin{aligned}
& d E_{y}\left(a^{+}\right) / d x=a_{11} E_{y}\left(a^{+}\right)+a_{12} \\
& d E_{z}\left(a^{+}\right) / d x=a_{21} E_{z}\left(a^{+}\right)+a_{22}
\end{aligned}
$$

where

$$
\begin{aligned}
& a_{11}=a_{21}=\lambda\left(\beta_{1} / \beta_{2}\right) \\
& a_{12}=\left(\beta_{2}^{2}-\beta_{1}^{2} / \beta_{2}\right) d E_{y}(d) / d x \\
& a_{22}=\left(\beta_{2}^{2}-\beta_{1}^{2} / \beta_{2}\right) d E_{z}(d) / d x
\end{aligned}
$$

The boundary conditions for the ODEs (18) at the plasma side of the plasma and vacuum interface $x=a^{-}$are still given by Eq. (93), but $a_{11}, a_{12}, a_{21}$, and $a_{22}$ are replaced by Eqs. (113)-(115).

\section{POWER ABSORPTION AND KINETIC ENERGY FLUX}

In an inhomogeneous plasma, the local power absorption $P_{\alpha}$ at $\mathbf{r}$ by species $\alpha$ can be defined as the time-average rate of change in the energy of the particles passing through $r$, and is equal to[20]

$$
P_{\alpha}(\mathbf{r})=(1 / 2) \operatorname{Re} \int d \mathbf{v}^{\prime}\left\langle q_{\alpha} \mathbf{E}\left(\mathbf{r}^{\prime}, t^{\prime}\right) \cdot \mathbf{v} f_{\alpha}^{*}\left(\mathbf{r}^{\prime}, \mathbf{v}^{\prime}, t^{\prime}\right)\right\rangle_{t^{\prime}}
$$

where $\langle\ldots\rangle_{t^{\prime}}$ denotes the time average over $t^{\prime}$ from $t^{\prime}=-\infty$ to $t^{\prime}=t, \mathrm{r}^{\prime}\left(t^{\prime}\right)=\mathrm{r}$ and $\mathbf{v}^{\prime}\left(t^{\prime}\right)=\mathbf{v}$ at $t^{\prime}=t$ describe the particle trajectory, $f_{\alpha}$ is the perturbed distribution function for species $\alpha$, and the asterisk indicates the complex conjugate. The right-hand side of Eq. (116) represents the average rate of work done by the $R F$ wave field on plasma particles. The definition of $P_{\alpha}$ [Eq. (116)] has some profound properties[21]: (a) it is positive definite if a plasma is in local thermal equilibrium; (b) it agrees with the well-known weak damping limit for a single mode in a homogeneous plasma; (c) it is consistent with Poynting's theorem [see Eq. (117) below]; and (d) superposition for different Fourier modes is valid in a planestratified plasma.

It can be proven that the difference between $P_{\alpha}$ and $(1 / 2) \operatorname{Re}\left(\mathbf{J}_{\alpha}^{*} \cdot \mathbf{E}\right)$ is the divergence of a vector[21]. Thus, for a 1-D slab model, 


$$
(1 / 2) \operatorname{Re}\left(\mathbf{J}_{\alpha}^{*} \cdot \mathbf{E}\right)=P_{\alpha}+\partial K_{\alpha} / \partial x,
$$

where $K_{\alpha}$ is the kinetic energy flux carried by coherent oscillation of particles in species $\alpha$. The total energy flux is the sum of the Poynting flux $S_{p x}=\hat{\mathbf{x}} \cdot \operatorname{Re}\left(\mathbf{E}^{*} \times \mathbf{B}\right) / 2 \mu_{0}$ and all kinetic energy fluxes $K_{\alpha}$,

$$
S_{x}=S_{p x}+\sum_{\alpha} K_{\alpha}
$$

where $\hat{\mathbf{x}}$ is the unit vector along the coordinate $x$. From the Poynting theorem,

$$
\frac{\partial S_{p x}}{\partial x}+\frac{1}{2} \operatorname{Re}\left(\mathrm{E}^{*} \cdot \sum_{\alpha} \mathrm{J}_{\alpha}\right)=0
$$

and Eqs. (117) and (118), we obtain

$$
\frac{\partial S_{x}}{\partial x}+\sum_{\alpha} P_{\alpha}=0
$$

\subsection{Power Absorption}

In ICRF, power absorption by electrons is due to electron Landau damping (ELD) and transit-time magnetic pumping (TTMP) and is determined by $n=0$ electron cyclotron harmonics. Using the definition of the power absorption (116) to calculate the power absorption up to the second order of the Larmor radius, we obtain for electrons[16]

$$
P_{e}^{(0)}=\frac{\sqrt{\pi} \epsilon_{0}}{2} \frac{\omega_{p e}^{2}}{\omega} \xi_{0 e} e^{-\xi_{0 e}^{2}}\left[2 \xi_{0 e}^{2}\left|E_{z}\right|^{2}+2 \frac{v_{e}}{\Omega_{e}} \omega \xi_{0 e} \operatorname{Im}\left(E_{z}^{*} B_{z}\right)+\frac{v_{e}^{2}}{\Omega_{e}^{2}} \omega^{2}\left|B_{z}\right|^{2}\right],
$$

where $B_{z}=-(1 / \omega)\left(i E_{y}^{\prime}+k_{y} E_{x}\right)$, and $\xi_{n \alpha}=\left(\omega-n \Omega_{\alpha}\right) / k_{z} v_{\alpha}$. In Eq. (121), the first term is ELD, the last term is electron TTMP, and the second term is a cross-term. ELD, the cross-term, and electron TTMP come from the zero, first, and second order of the Larmor radius expansion, respectively. Note that $P_{e}^{(0)}$ from Eq. (121) is positive definite.

For ions in ICRF, Landau damping and TTMP are very small and can be neglected, and we need to calculate only harmonic resonance absorption. From Eq. (116), we find that the power absorption due to the ion cyclotron resonance is[16]

$$
\begin{aligned}
P_{i}^{(1)}= & \frac{\sqrt{\pi} \epsilon_{0}}{4} \frac{\omega_{p i}^{2}}{\omega} \xi_{0 i} e^{-\xi_{1 i}^{2}}\left|E_{+}\right|^{2} \\
& +\frac{\sqrt{\pi} \epsilon_{0}}{8} \frac{\omega_{p i}^{2}}{\omega} \frac{v_{i}^{2}}{\Omega_{i}^{2}} \xi_{0 i} e^{-\xi_{1 i}^{2} \operatorname{Re}}\left\{E_{+}^{*}\left[2 E_{+}^{\prime \prime}-E_{-}^{\prime \prime}+2 k_{y} E_{-}^{\prime}-k_{y}^{2}\left(2 E_{+}+E_{-}\right)\right]\right\} \\
& +\frac{\sqrt{\pi} \epsilon_{0}}{8} \frac{d^{2}}{d x^{2}}\left(\frac{\omega_{p i}^{2}}{\omega} \frac{v_{i}^{2}}{\Omega_{i}^{2}} \xi_{0 i} e^{-\xi_{1 i}^{2}}\left|E_{+}\right|^{2}\right) \\
& +\frac{\sqrt{\pi} \epsilon_{0}}{8} k_{y} \frac{\Omega_{i}}{\omega} \frac{d}{d x}\left(-\frac{\omega_{p i}^{2}}{\omega} \frac{v_{i}^{2}}{\Omega_{i}^{2}} \xi_{0 i} e^{-\xi_{1 i}^{2}}\right)\left|E_{+}\right|^{2}
\end{aligned}
$$

where $E_{ \pm}=E_{x} \pm i E_{y}$, and the primes represent derivatives with respect to $x$. The first term in Eq. (122) comes from the zero-order Larmor radius expansion and is the same as that in 
a uniform plasma. The second term comes from the second-order Larmor radius expansion. It can be shown that it reduces to the correct form in the uniform plasma limit[18]. Note that the zero-order term in Eq. (122) is positive definite, but the second order term is not explicitly positive definite. However, the total $P_{i}^{(1)}$ should be positive within the error of the finite Larmor radius expansion.

The power absorption due to the second-harmonic resonance is[16]

$$
P_{i}^{(2)}=\frac{\sqrt{\pi} \epsilon_{0}}{8} \frac{\omega_{p i}^{2}}{\omega} \frac{v_{i}^{2}}{\Omega_{i}^{2}} \xi_{0 i} e^{-\xi_{2 i}^{2}}\left|E_{+}^{\prime}-k_{y} E_{+}\right|^{2},
$$

which comes from the second-order Larmor radius expansion and is positive definite. The power absorption $P_{i}^{(-1)}$ and $P_{i}^{(-2)}$ is negligibly small for ICRF since $P_{i}^{(n)}$ has a factor of $\operatorname{Im}\left[Z\left(\xi_{n i}\right)\right]=\sqrt{\pi} \exp \left(-\xi_{n i}^{2}\right)$.

\subsection{Kinetic Energy Flux}

The kinetic energy flux is found by subtracting $P_{\alpha}$ from $(1 / 2) \operatorname{Re}\left(\mathrm{J}_{\alpha}^{*} \cdot \mathrm{E}\right)$. For electrons,

$$
K_{e}^{(0)}=-\frac{\epsilon_{0}}{2} \frac{\omega_{p e}^{2}}{\omega} \frac{v_{e}}{\Omega_{e}} \xi_{0 e} \operatorname{Im}\left[\frac{v_{e}}{\Omega_{e}} Z\left(\xi_{0 e}\right)\left(E_{y}^{\prime}-i k_{y} E_{x}\right) E_{y}^{*}+\frac{1}{2} Z^{\prime}\left(\xi_{0 e}\right) E_{z} E_{y}^{*}\right]
$$

where the prime on the plasma dispersion function $Z\left(\xi_{n \alpha}\right)$ indicates the derivative with respect to its argument $\xi_{n \alpha}$. The kinetic energy flux corresponding to the ion cyclotron absorption $P_{i}^{(1)}$ is

$$
\begin{aligned}
K_{i}^{(1)}=-\frac{\epsilon_{0}}{16} & \frac{\omega_{p i}^{2}}{\omega} \frac{v_{i}^{2}}{\Omega_{i}^{2}} \xi_{0 i} \operatorname{Im}\left[Z\left(\xi_{1 i}\right)\left(4 E_{+} E_{+}^{\prime *}+E_{+}^{\prime} E_{-}^{*}-E_{+} E_{-}^{\prime *}-2 k_{y} E_{+} E_{-}^{*}\right)\right] \\
& -\operatorname{Im}\left\{\frac{d}{d x}\left[\frac{\epsilon_{0}}{16} \frac{\omega_{p i}^{2}}{\omega} \frac{v_{i}^{2}}{\Omega_{i}^{2}} \xi_{0 i} Z\left(\xi_{1 i}\right)\right] E_{+} E_{-}^{*}\right\}
\end{aligned}
$$

The kinetic energy flux corresponding to the second harmonic absorption $P_{i}^{(2)}$ is

$$
K_{i}^{(2)}=-\frac{\epsilon_{0}}{8} \frac{\omega_{p i}^{2}}{\omega} \frac{v_{i}^{2}}{\Omega_{i}^{2}} \xi_{0 i} \operatorname{Im}\left[Z\left(\xi_{2 i}\right)\left(E_{+}^{\prime}-k_{y} E_{+}\right) E_{+}^{*}\right] .
$$

Unlike $P_{\alpha}^{(n)}, K_{\alpha}^{(n)}$ has both $\operatorname{Im}\left[Z\left(\xi_{n \alpha}\right)\right]$ and $\operatorname{Re}\left[Z\left(\xi_{n \alpha}\right)\right]$. Near the resonance, $K_{\alpha}^{(n)}$ is dominated by terms having a factor of $\operatorname{Im}\left[Z\left(\xi_{n \alpha}\right)\right]=\sqrt{\pi} \exp \left(-\xi_{n \alpha}^{2}\right)$, whereas away from the resonance, $K_{\alpha}^{(n)}$ is dominated by terms having a factor of $\operatorname{Re}\left[Z\left(\xi_{n \alpha}\right)\right] \approx-\left(1 / \xi_{n \alpha}\right)$, which has the same order of magnitude for $n$ and $-n$. Therefore, we need to retain the terms having $\operatorname{Re}\left[Z\left(\xi_{n \alpha}\right)\right]$ in $K_{\alpha}^{(-1)}$ and $K_{\alpha}^{(-2)}$, although the power absorption $P_{\alpha}^{(-1)}$ and $P_{\alpha}^{(-2)}$ is negligibly small. The kinetic energy flux for $n=-1$ is

$$
\begin{gathered}
K_{i}^{(-1)=\frac{\epsilon_{0}}{16}} \frac{\omega_{p i}^{2}}{\omega} \frac{v_{i}^{2}}{\Omega_{i}^{2}} \frac{\xi_{0 i}}{\xi_{-1 i}} \operatorname{Im}\left(4 E_{-} E_{-}^{\prime *}+E_{-}^{\prime} E_{+}^{*}-E_{-} E_{+}^{\prime *}+2 k_{y} E_{-} E_{+}^{*}\right) \\
+\frac{d}{d x}\left(\frac{\epsilon_{0}}{16} \frac{\omega_{p i}^{2}}{\omega} \frac{v_{i}^{2}}{\Omega_{i}^{2}} \frac{\xi_{0 i}}{\xi_{-1 i}}\right) \operatorname{Im}\left(E_{+} E_{-}^{*}\right) .
\end{gathered}
$$

The kinetic energy flux for $n=-2$ is 


$$
K_{i}^{(-2)}=\frac{\epsilon_{0}}{8} \frac{\omega_{p i}^{2}}{\omega} \frac{v_{i}^{2}}{\Omega_{i}^{2}} \frac{\xi_{0 i}}{\xi_{-2 i}} \operatorname{Im}\left(E_{-}^{\prime} E_{-}^{*}\right) .
$$

However, the ion kinetic energy flux near the ion resonance is still much larger than the ion kinetic energy flux away from the resonance. An estimate can be found from

$$
\frac{\operatorname{Im}[Z(0)]}{\operatorname{Re}\left[Z\left(\Omega_{i} / k_{z} v_{i}\right)\right]} \approx-\sqrt{\pi}\left(\Omega_{i} / k_{z} v_{i}\right)
$$

which is usually very large for present ICRF experiments. Of course, this is a very rough estimate, and a detailed study of their contribution to the kinetic energy flux needs to include the radial spatial variation of the $R F$ electric field and requires numerical computation. For example, near a two-ion hybrid resonance, $\operatorname{Im}\left[Z\left(\xi_{1 \alpha}\right)\right]=\sqrt{\pi} \exp \left(-\xi_{1 i}^{2}\right)$ may not be very small, and the spatial variation of the electric field is strong due to mode conversion. In that case, $K_{i}^{(1)}$ may contribute significantly to the kinetic energy flux of ions.

\section{NUMERICAL METHOD}

Since solving ODEs (18) with six boundary conditions is a standard two-point value problem, we use a routine (D02GBF) in the Numerical Algorithm Group (NAG) Fortran Library[22], which solves a general linear two-point boundary value problem for a system of $N$ real first-order ODEs. The set of $N$ real ODEs are written in the form of

$$
\frac{d y}{d x}=F(x) y+G(x)
$$

in the range of $x=\left(x^{-}, x^{+}\right)$with the boundary conditions

$$
C y\left(x^{-}\right)+D y\left(x^{+}\right)=\gamma .
$$

Here, $F(x), C$, and $D$ are $N \times N$ real matrices, and $y, G(x)$, and $\gamma$ are $N$-component real vectors. To use routine D02GBF, we convert the six complex ODEs (18) with the six complex boundary conditions to a set of 12 real ODEs in the form of Eq. (130) with 12 real boundary conditions in the form of Eq. (131).

The routine uses a finite difference method with a deferred correction. An absolute error tolerance and an initial mesh need to be supplied. The routine constructs a solution on a mesh defined by adding more points to the initial mesh. The solution is chosen so that the error is everywhere less than the tolerance and the error is approximately equidistributed on the final mesh. GLOSI is written in Fortran 90, which is a new standard Fortran and has many excellent features of portability and easy maintenance[23]. The code consists of many subroutines and is maintained by the UNIX "make" utility.

\section{NUMERICAL RESULTS}

In this section, we give several examples to demonstrate that the code can be used to find local and global solutions for RF plasma heating. The first example is a 1-D modeling for the TFTR mode conversion heating experiment. We solve the wave equation locally around the two-ion hybrid resonance. The second example is the JET relevant minority ion heating. We solve the wave equation globally in a region spanning from one conducting 
wall to the other conducting wall. The last example uses the fixed tangential magnetic field boundary conditions at the edge of the plasma and the scattering boundary conditions at the other end to find the impedance matrix.

The first example is TFTR Shot 73774 performed in 1994. The fast wave excited by an antenna is mode-converted to the ion Bernstein wave at the two-ion hybrid resonance. The short wavelength IBW is absorbed by electrons within a small spatial interval, which produces localized electron heating and current drive. The toroidal magnetic field $B_{0}=$ $4.44 \mathrm{~T}$ at the major radius $R_{0}=2.62 \mathrm{~m}$ and the minor radius $a=0.96 \mathrm{~m}$. The plasma consists of $50 \%{ }^{3} \mathrm{He}$ and $50 \%{ }^{4} \mathrm{He}$. The peak electron density is $n_{0}=4.0 \times 10^{19} \mathrm{~m}^{-3}$. The peak temperature for electrons is $4.2 \mathrm{keV}$, and the peak temperature for ions is $2.8 \mathrm{keV}$. The density and temperature profiles are assumed to be parabolic and Gaussian, respectively,

$$
\begin{aligned}
& n(x)=n_{0}\left(1-x^{2} / a^{2}\right), \\
& T(x)=T_{0} \exp \left(-3 x^{2} / a^{2}\right) .
\end{aligned}
$$

Figure 1 shows some of the results given by the code for the parallel wave number $k_{z}=$ $10 \mathrm{~m}^{-1}$ and the RF wave frequency $\omega / 2 \pi=43 \mathrm{MHz}$. In calculation, we impose the scattering boundary conditions at $x=-0.31 \mathrm{~m}$ and $x=-0.16 \mathrm{~m}$ with $x=0$ at the major radius of the tokamak. The boundary conditions at $x=-0.16 \mathrm{~m}$ represent incident and reflected fast waves from the low field side and outgoing ion Bernstein and shear Alfvén waves. The boundary conditions at $x=-0.30 \mathrm{~m}$ represent a transmitted fast wave and outgoing ion Bernstein and shear Alfvén waves. The solution is normalized by imposing $E_{y}=1 \mathrm{~V} / \mathrm{m}$ at $x=-0.16 \mathrm{~m}$. Due to the strong electron absorption, the mode-converted IBW is almost fully absorbed before it reaches $x=-0.31 \mathrm{~m}$. For this case, $18.2 \%$ of the incident fast wave power is reflected and about $55.7 \%$ is transmitted. The rest of the power $26.1 \%$ is absorbed by electrons, and ion absorption is negligibly small. Figure 1 (e) checks whether the solutions satisfy the Poynting theorem (119). It plots $\partial S_{p x} / \partial x, \operatorname{Re}\left(\mathbf{E}^{*} \cdot \sum_{\alpha} \mathbf{J}_{\alpha}\right) / 2$, and $\partial S_{p x} / \partial x+\operatorname{Re}\left(\mathbf{E}^{*} \cdot \sum_{\alpha} \mathrm{J}_{\alpha}\right) / 2$ vs $x$. Figure 2 shows the dependence of the power absorption, reflection, transmission, and mode conversion coefficients on the parallel wave number $k_{z}$. The sum of the power absorption, reflection, transmission, and mode conversion coefficients must be equal to unity to satisfy the power conservation.

The second example is for the parameters relevant to JET minority ion heating. We solve the wave equation globally between two conducting walls at $x=-1.35 \mathrm{~m}$ and $x=1.35 \mathrm{~m}$. An antenna is located at $x=1.30 \mathrm{~m}$. To excite a fast wave in the plasma, we take the antenna current density to be in the poloidal direction, i.e., $\mathrm{J}_{\text {ext }}=\hat{\mathbf{y}} \delta(x-1.30) \mathrm{A} / \mathrm{m}^{2}$. The plasma has the minor radius $a=1.25 \mathrm{~m}$ and the toroidal magnetic field $B_{0}=3.45 \mathrm{~T}$ at the major radius $R_{0}=2.96 \mathrm{~m}$. The plasma consists of $90 \%$ deuterium and $10 \%$ hydrogen. The peak electron density is $n_{0}=5.0 \times 10^{19} \mathrm{~m}^{-3}$. The peak temperature for all species is $3.0 \mathrm{keV}$. The density and temperature profiles are still given by Eqs. (132) and (133), respectively. Figure 3 shows some of the results given by the code for the parallel wave number $k_{z}=6.0 \mathrm{~m}^{-1}$ and the $\mathrm{RF}$ wave frequency $\omega / 2 \pi=52.4 \mathrm{MHz}$. In Fig. 3 , we only show profiles within the plasma, but not the analytical solutions in the vacuum. Since the plasma and the antenna are confined within perfect conducting walls, the total power emitted by the antenna is fully absorbed by the plasma. The electrons absorb $4.0 \%$ of the power, the major ion species deuterium absorbs $2.9 \%$, and the minority ion hydrogen absorbs the remaining $93.1 \%$. 
The last example is to calculate the impedance matrix for TFTR for a pair of toroidal and poloidal modes. We impose the boundary conditions $B_{y}=1 \mathrm{~T}$ and $B_{z}=0$ or $B_{y}=0$ and $B_{z}=1 \mathrm{~T}$ right outside the plasma edge of the low field side $x=0.96 \mathrm{~m}$. We also impose the scattering boundary conditions at $x=0.86 \mathrm{~m}$, which allows a transmitted fast wave and outgoing ion Bernstein and shear Alfvén waves. We take the temperature profile as given by Eq. (133). To better model the density profile, we take different profiles in the core region and scrape-off region. In the core, we still use Eq. (132) for the density profile. In the scrape-off region, we assume that the density profile exponentially decays:

$$
n(x)=n_{s} \exp \left[-\left(x-x_{s}\right) / L_{n}\right]
$$

where $n_{s}$ is the density at the separatrix $x_{s}=0.90 \mathrm{~m}$, and the fall-off length $L_{n}=0.03 \mathrm{~m}$. We also assume that the plasma consists of deuterium only, and other parameters are the same as those in the first example. For $k_{z}=7.0 \mathrm{~m}^{-1}$ and $k_{y}=0 \mathrm{~m}^{-1}$, we find that the impedance matrix is

$$
\mathcal{Z}=\left[\begin{array}{cc}
1.15-i 1.04 & -29.7+i 31.7 \\
0.407-i 5.68 & -1.13+i 1.06
\end{array}\right] \text { ohm }
$$

We can calculate the impedance matrices for a whole spectrum of the toroidal and poloidal modes, which can be used to calculate antenna loading by an antenna code[14].

\section{CONCLUSION}

In this report, we present a numerical code GLOSI which can solve the 1-D wave equation for the ICRF plasma heating. The code solves the sixth-order equation, which allows finite parallel wave electric field $E_{z}$, so that the code can be used in regions with either an high or low plasma density. One important feature of the code is that it can be used to find either global or local solutions when coupled with appropriate boundary conditions at the two ends. The code has choices of three different types of boundary conditions which cover various physical situations in ICRF plasma heating.

\section{ACKNOWLEDGMENTS}

The work of C. Y. Wang is supported by an appointment to the Oak Ridge National Laboratory Postdoctoral Research Associates Program, administered jointly by the Oak Ridge Institute for Science and Education and the Oak Ridge National Laboratory. The work of D. B. Batchelor, E. F. Jaeger, and M. D. Carter is supported by the U.S. Department of Energy under Contract No. DE-AC05-840R21400 with Martin Marietta Energy Systems, Inc. 


\section{REFERENCES}

[1] M. Murakami et al., in AIP Conference Proceedings 289: Radio Frequency Power in Plasmas, Boston, MA, 1993, edited by M. Porkolab and J. Hosea (American Institute of Physics, New York, 1994), p. 48.

[2] J. Jacquinot, V. P. Bhatnagar, C. Gormezano, and the JET Team, Plasma Phys. Control. Fusion 35, A35 (1993).

[3] S. C. Chiu and T. K. Mau, Nucl. Fusion 23, 1613 (1983).

[4] P. L. Colestock and R. J. Kashuba, Nucl. Fusion 23, 763 (1983).

[5] A. Fukuyama, K. Itoh, and S.-I. Itoh, Comput. Phys. Rep. 4, 137 (1986).

[6] H. Romero and J. Scharer, Nucl. Fusion 27, 363 (1987).

[7] E. F. Jaeger, D. B. Batchelor, and H. Weitzner, Nucl. Fusion 28, 53 (1988).

[8] I. R. Shokair, R. W. Conn, and T. K. Mau, Nucl. Fusion 28, 1393 (1988).

[9] M. Brambilla, Nucl. Fusion 28, 549 (1988).

[10] C. Y. Wang, E. F. Jaeger, D. B. Batchelor, and K. L. Sidikman, Phys. Plasmas 1, 3890 (1994).

[11] D. C. Stallings, D. B. Batchelor, C. Y. Wang, and M. D. Carter, Bull. Am. Phys. Soc. 39, 1628 (1994).

[12] R. Majeski, C. K. Phillips, and J. R. Wilson, Phys. Rev. Lett. 73, 2204 (1994).

[13] R. S. Sund and J. E. Scharer, Phys. Fluids B 3, 1326 (1991).

[14] M. D. Carter, D. B. Batchelor, and D. C. Stallings, ORNL/TM-12464, Oak Ridge National Laboratory, September 1993.

[15] C. Y. Wang, M. D. Carter, D. B. Batchelor, and E. F. Jaeger, ORNL/TM-12721, Oak Ridge National Laboratory, April 1994.

[16] M. Brambilla, Plasma Phys. Control. Fusion 31, 723 (1989).

[17] B. D. Fried and S. D. Conte, The Plasma Dispersion Function, Academic Press, Inc., New York, 1961.

[18] T. H. Stix, Waves in Plasmas, American Institute of Physics, New York, 1992.

[19] J. A. Heikkinen, T. Hellsten, and M. J. Alava, Nucl. Fusion 31, 417 (1991).

[20] B. D. McVey, R. S. Sund, and J. E. Scharer, Phys. Rev. Lett. 55, 507 (1985).

[21] M. Brambilla and T. Krucken, Plasma Phys. Control. Fusion 30, 1083 (1988).

[22] The NAG Fortran Library Manual (NAG Inc., 1400 Opus Place, Suite 200, Downers Grove, IL 60515, 1993), Mark 16. 
[23] J. C. Adams, W. S. Brainerd, J. T. Martin, B. T. Smith, and J. L. Wagener, Fortran 90 Handbook, Intertext Publications/Multiscience Press, Inc., and McGraw-Hill Book Company, New York, 1992. 

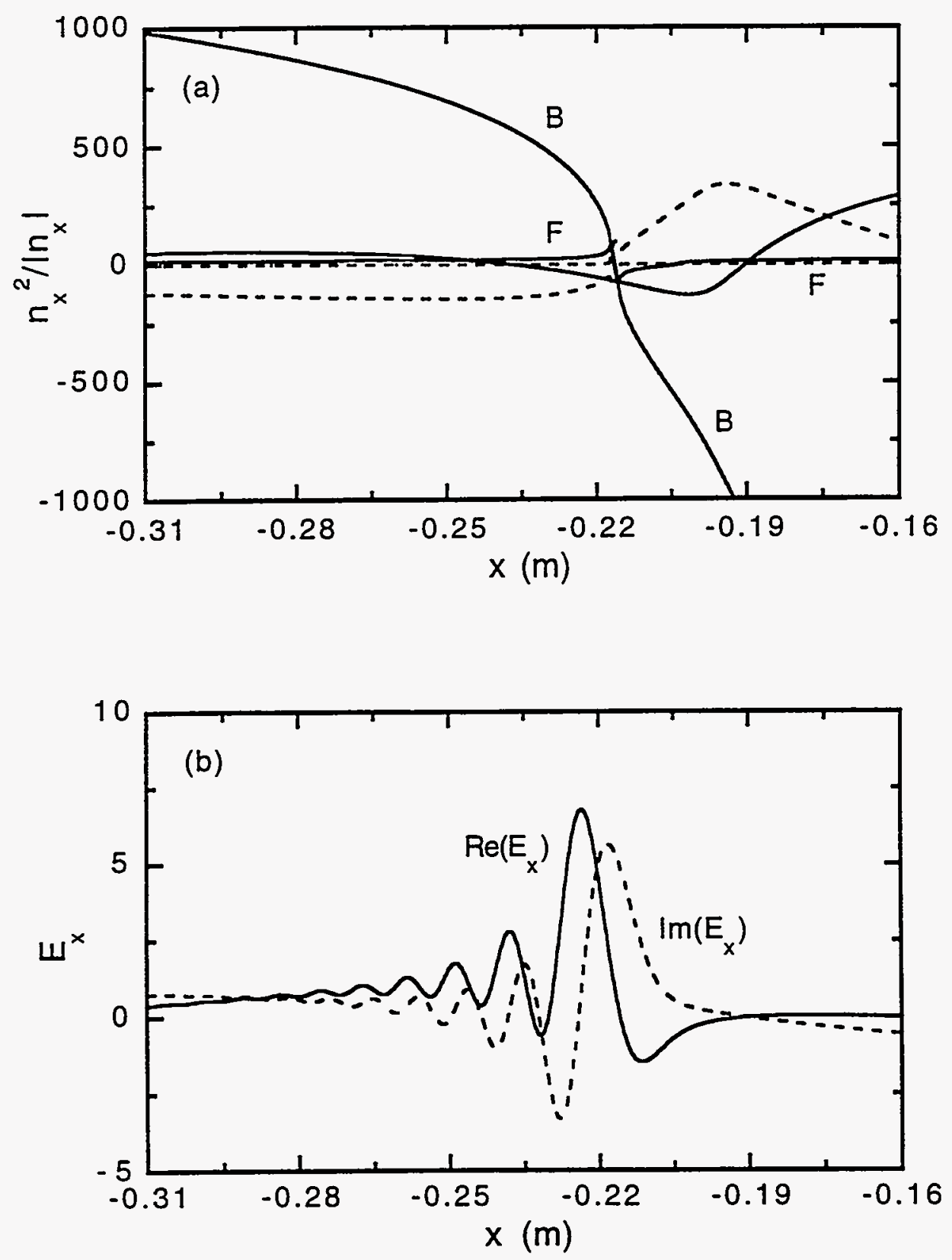

Figure 1: Results obtained by GLOSI for the TFTR Shot 73774 parameters. Here, $k_{z}=$ $10 \mathrm{~m}$ and $\omega / 2 \pi=43 \mathrm{MHz}$. (a) Real part (solid) and imaginary part (dash) of $n_{x}^{2} /\left|n_{x}\right|$ vs $x$, where $n_{x}=k_{x} c / \omega$. Ion Bernstein waves are denoted by $B$ and fast waves are denoted by $F$. (b) $E_{x}$ vs $x$. (c) $E_{y}$ vs $x$. (d) $E_{z}$ vs $x$ (e) Terms in Poynting theorem. (f) Total energy flux $\left(-S_{x}\right)$ and electron power absorption $\left(P_{e}\right)$ vs $x$. 

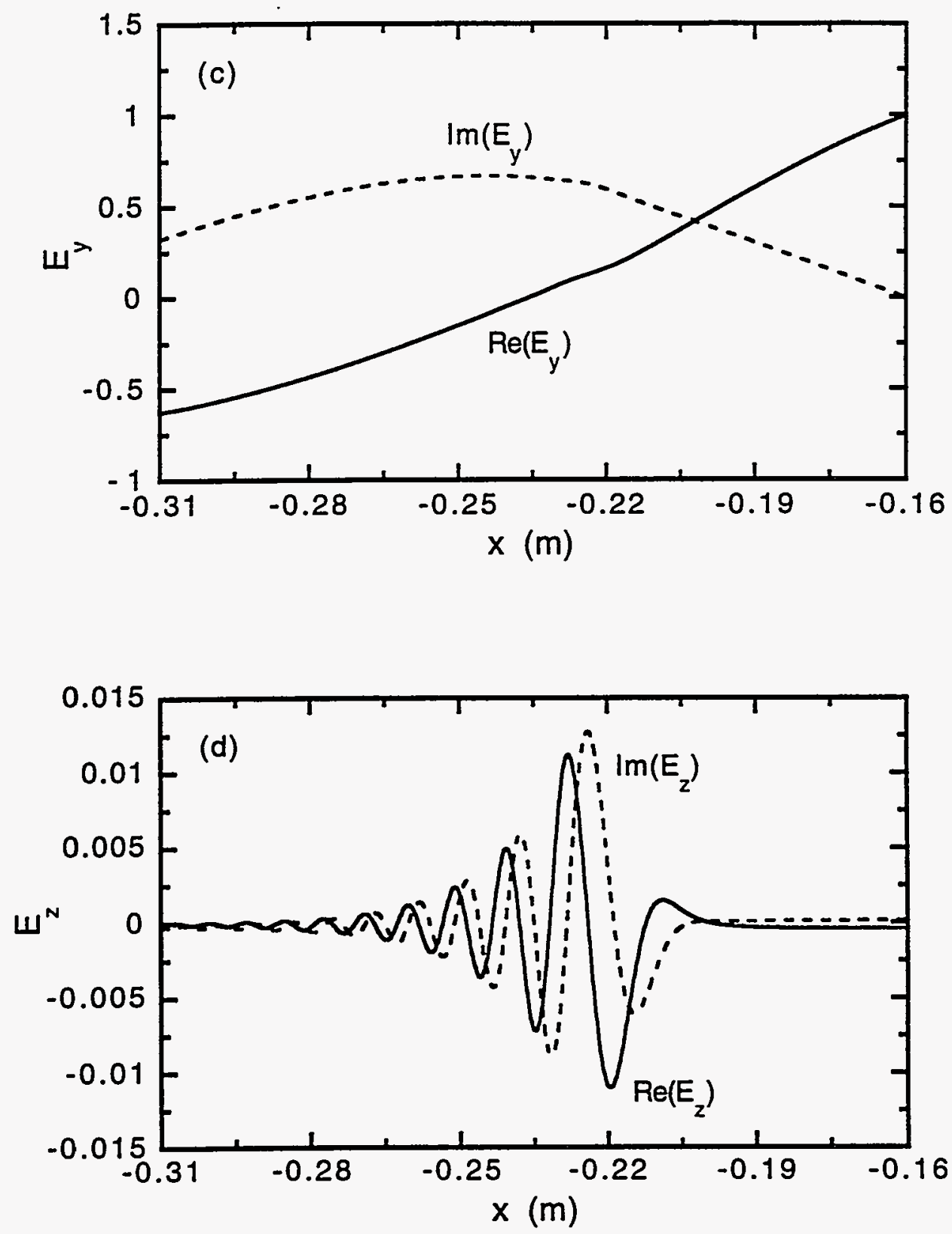

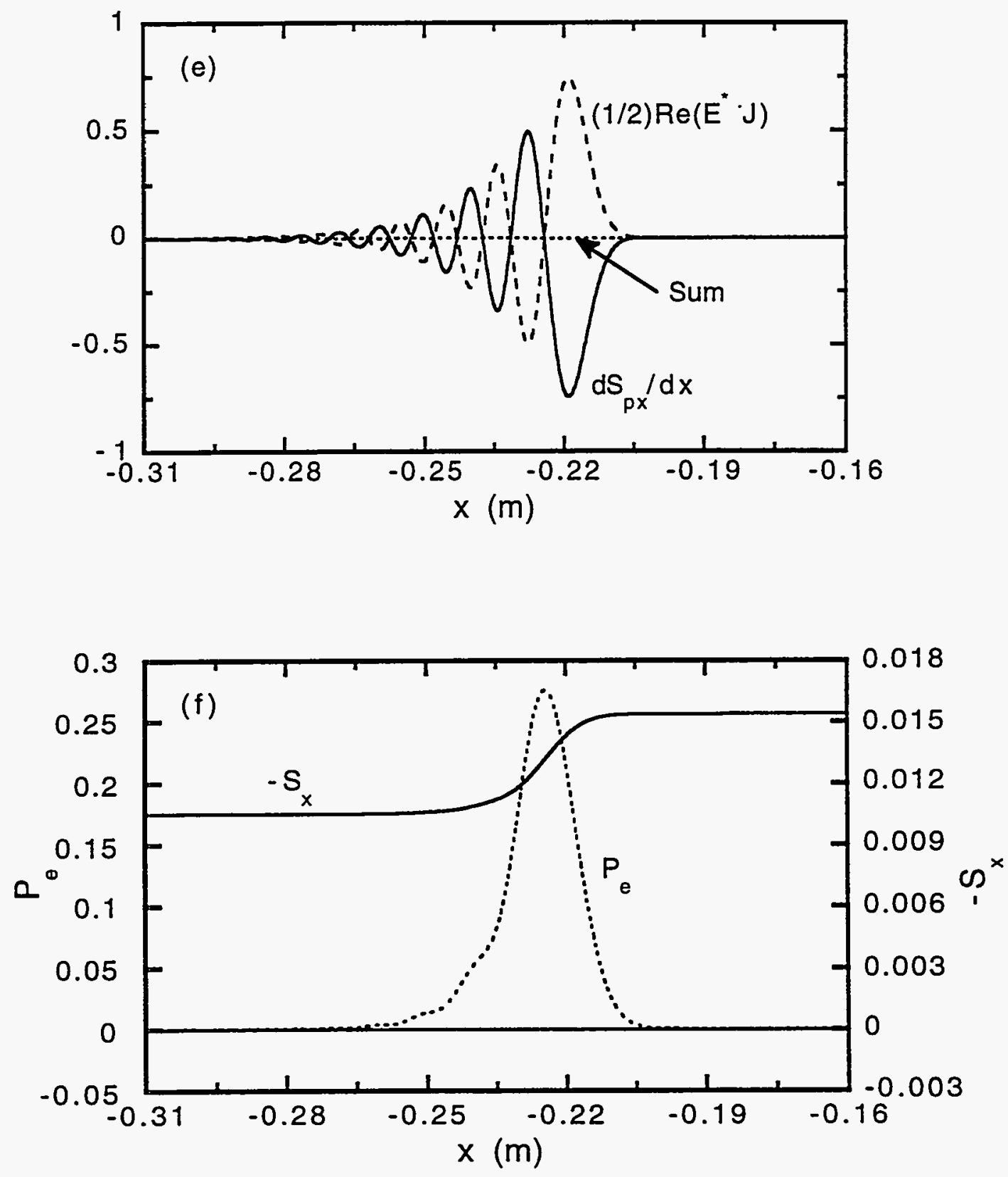


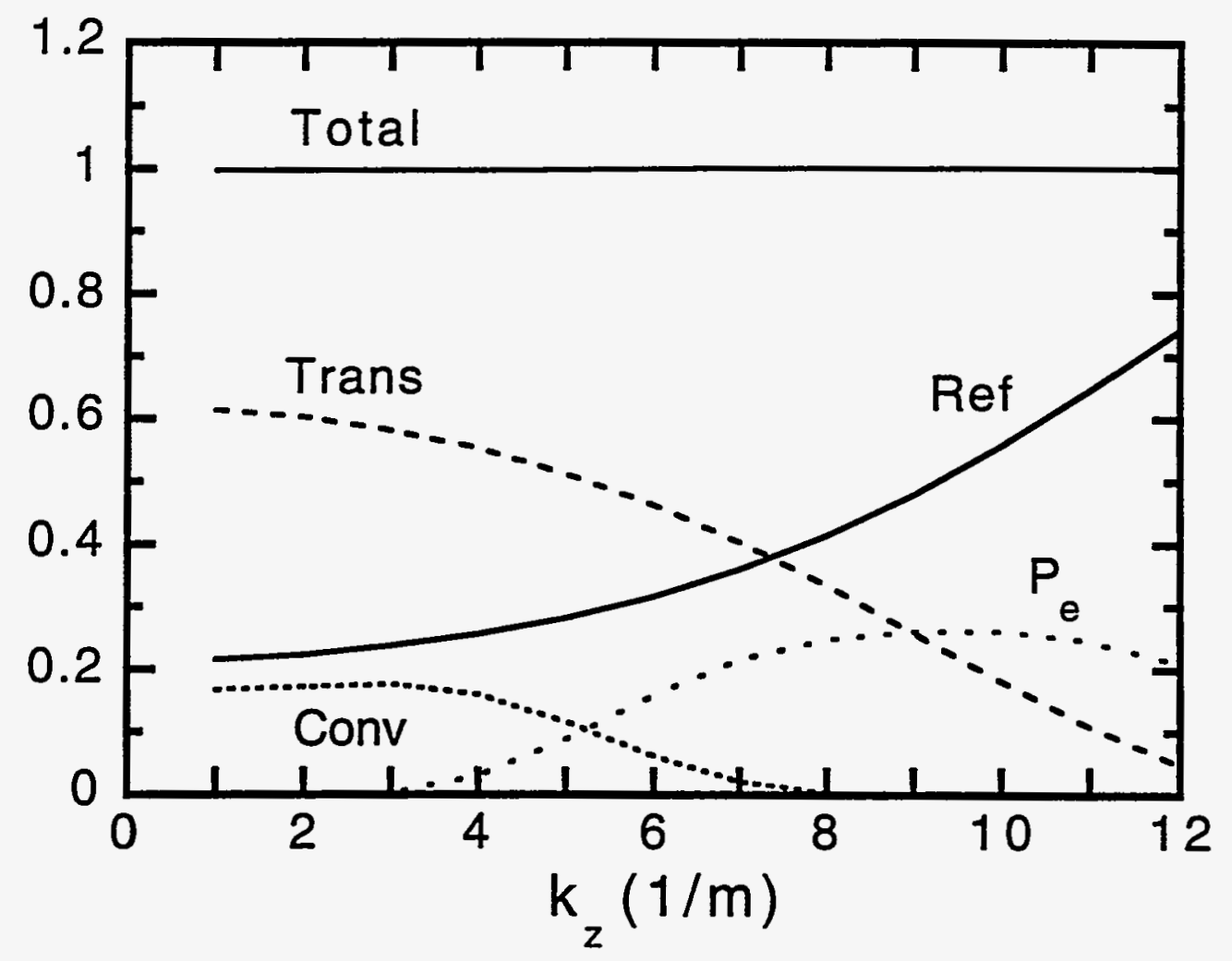

Figure 2: Transmission, reflection, mode conversion, and electron power absorption coefficients vs $k_{z}$. Absorption by the ion species is negligible. 

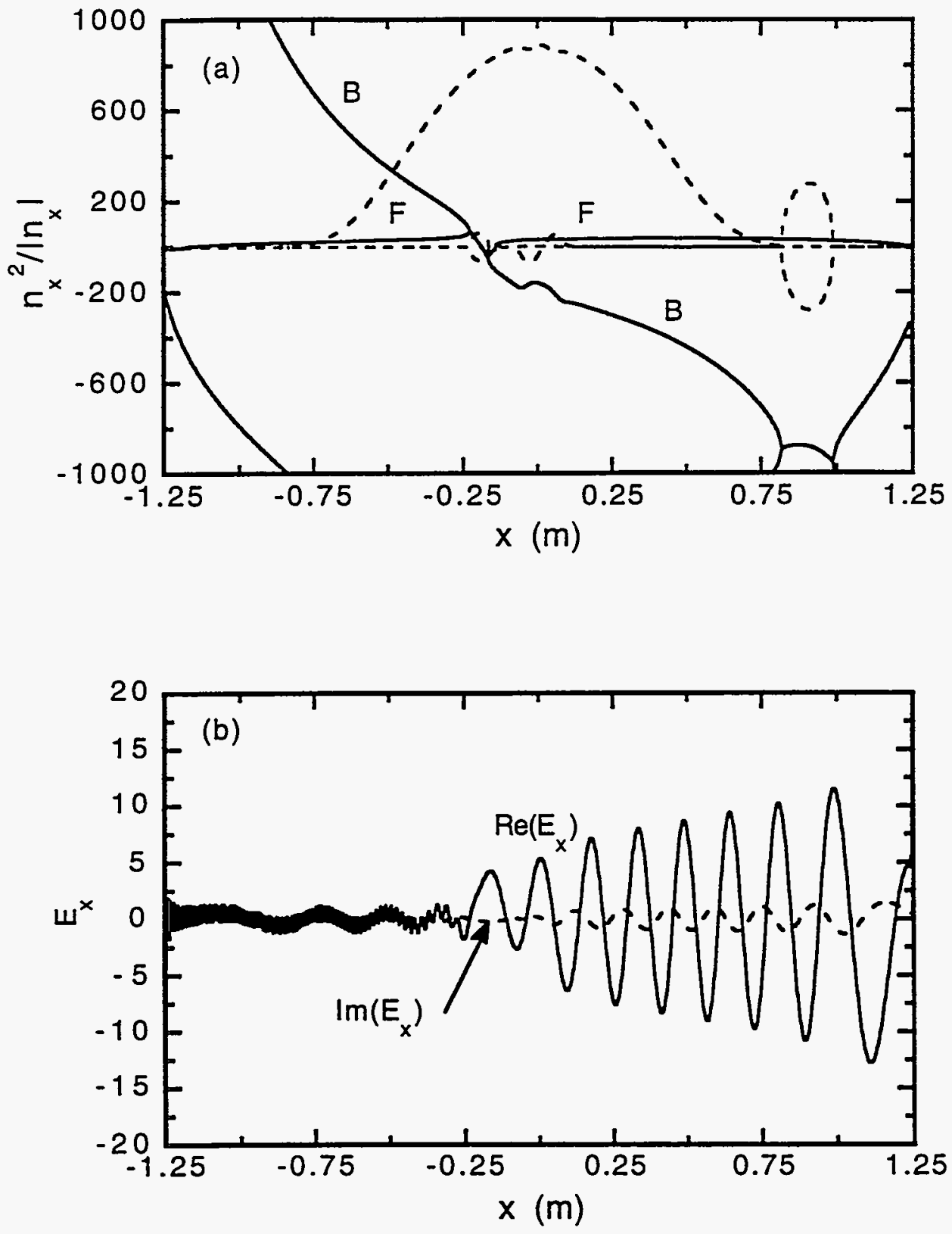

Figure 3: Results obtained by GLOSI for JET relevant minority hydrogen heating. Here, $k_{z}=6.0 \mathrm{~m}$ and $\omega / 2 \pi=52.4 \mathrm{MHz}$. (a) Real part (solid) and imaginary part (dash) of $n_{x}^{2} /\left|n_{x}\right|$ vs $x$. Ion Bernstein waves are denoted by $B$ and fast waves are denoted by $F$. (b) $E_{x}$ vs $x$. (c) $E_{y}$ vs $x$. (d) $E_{z}$ vs $x$. (e) Terms in Poynting theorem. (f) Total energy flux $\left(-S_{x}\right)$ and power absorbed by electrons $\left(P_{e}\right)$, majority ions $\left(P_{D}\right)$, and minority ions $\left(P_{H}\right)$ vs $x$. 

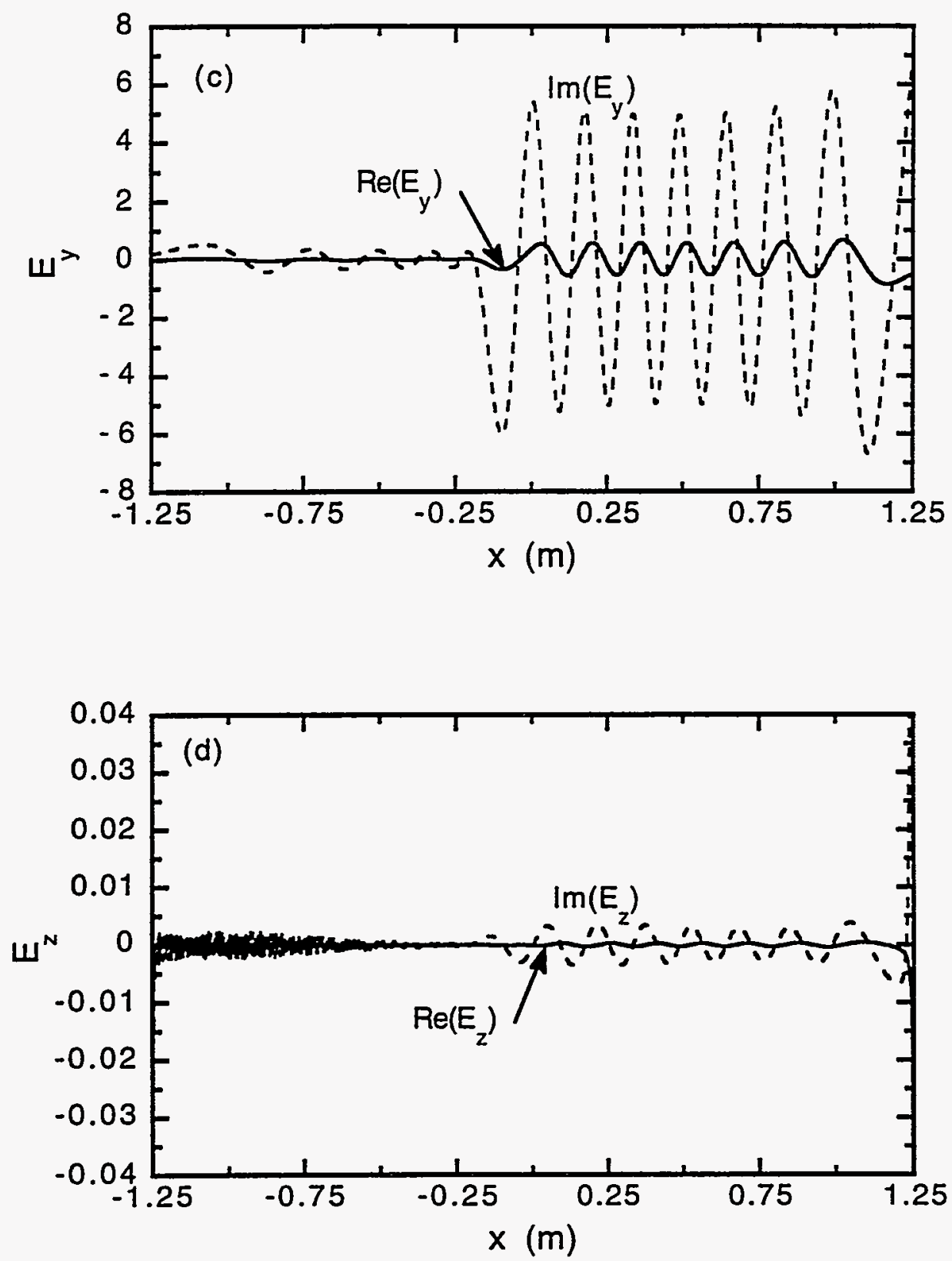

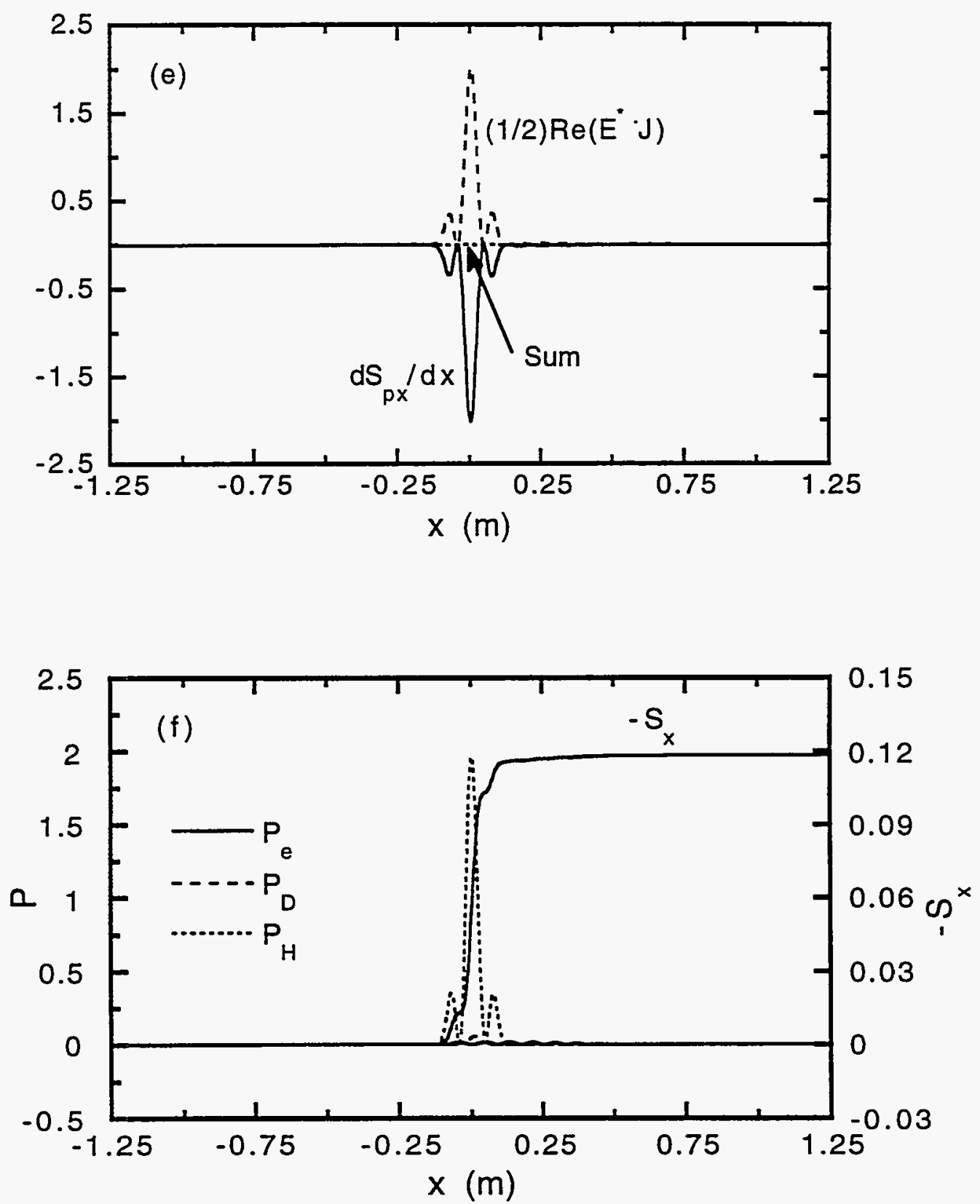
ORNL/TM-12923

Dist. Category UC-427

\section{INTERNAL DISTRIBUTION}

1. Director, ORNL Fusion Energy Division

2-6. D. B. Batchelor

7. L. A. Berry

8. B. A. Carreras

9. H. E. Clark

10. R. J. Colchin

11. F. E. Gethers

12. S. L. Milora

13. W. L. Stirling

14-15. Laboratory Records Department

16. Laboratory Records, ORNL-RC

17-18. Central Research Library

19. Document Reference Section

20. Fusion Energy Division Library

21-22. ET/FE Division Publications Office

23. ORNL Patent Office
24. F. W. Baity

25. T. S. Bigelow

26. M. D. Carter

27. R. C. Goldfinger

28. R. H. Goulding

29. S. P. Hirshman

30. D. J. Hoffman

31. W. A. Houlberg

32. E. F. Jaeger

33. D. A. Rasmussen

34. P. M. Ryan

35. D. C. Stallings

36. D. W. Swain

37-43. C. Y. Wang

44. J. H. Whealton

45. J. J. Yugo

\section{EXTERNAL DISTRIBUTION}

46. Office of the Assistant Manager for Energy Research and Development, Department of Energy, Oak Ridge Operations, P.O. Box 2000, Oak Ridge, TN 37831

47. N. A. Davies, Director, Office of Fusion Energy, Office of Energy Research, ER-50 Germantown, U.S. Department of Energy, Washington, DC 20545

48. M. Roberts, International Programs, Office of Fusion Energy, Office of Energy Research, ER-52 Germantown, U.S. Department of Energy, Washington, DC 20545

49. D. E. Baldwin, Lawrence Livermore National Laboratory, P.O. Box 5511, Livermore, CA 94550

50. R. W. Conn, Mechanical, Aerospace, and Nuclear Engineering Department, 6291 Boelter Hall, University of California, Los Angeles, CA 90024-1597

51. P. C. Liewer, MS 138-208, Jet Propulsion Laboratory, 4800 Oak Grove Drive, Pasadena, CA 91109

52. D. Sigmar, Plasma Fusion Center, Massachusetts Institute of Technology, 167 Albany St., NW16-288, Cambridge, MA 02139

53. K. I. Thomassen, L-637, Lawrence Livermore National Laboratory, P.O. Box 5511, Livermore, CA 94550

54. J. D. Callen, Department of Nuclear Engineering, University of Wisconsin, Madison, WI 53706-1687

55. S. O. Dean, Fusion Power Associates, Inc., 2 Professional Drive, Suite 248, Gaithersburg, MD 20879

56. H. K. Forsen, Bechtel Group, Inc., Research Engineering, P.O. Box 3965, San Francisco, CA 94119 
57. R. W. Gould, Department of Applied Physics, California Institute of Technology, Pasadena, CA 91125

58. R. A. Gross, Plasma Research Laboratory, Columbia University, New York, NY 10027

59. R. J. Hawryluk, Plasma Physics Laboratory, Princeton University, P.O. Box 451, Princeton, NJ 08543

60. D. M. Meade, Plasma Physics Laboratory, Princeton University, P.O. Box 451, Princeton, NJ 08543

61. W. M. Stacey, School of Nuclear Engineering and Health Physics, Georgia Institute of Technology, Atlanta, GA 30332

62. D. Steiner, Nuclear Engineering Department, NES Building, Tibbetts Avenue, Rensselaer Polytechnic Institute, Troy, NY 12181

63. R. Varma, Physical Research Laboratory, Navrangpura, Ahmedabad 380009, India

64. Bibliothek, Max-Planck Institut für Plasmaphysik, Boltzmannstrasse 2, D-8046 Garching, Federal Republic of Germany

65. Bibliothek, Institut für Plasmaphysik, KFA Jülich GmbH, Postfach 1913, D-5170 Jülich, Federal Republic of Germany

66. Bibliothek, KfK Karlsruhe GmbH, Postfach 3640, D-7500 Karlsruhe 1, Federal Republic of Germany

67. Bibliotheque, Centre de Recherches en Physique des Plasmas, Ecole Polytechnique Fédérale de Lausanne, 21 Avenue des Bains, $\mathrm{CH}-1007$ Lausanne, Switzerland

68. R. Aymar, CEN/Cadarache, Departement de Recherches sur la Fusion Contrôlée, F-13108 Saint-Paul-lez-Durance Cedex, France

69. Bibliothèque, CEN/Cadarache, F-13108 Saint-Paul-lez-Durance Cedex, France

70. Library, JET Joint Undertaking, Abingdon, Oxfordshire OX14 3EA, England

71. Library, FOM-Instituut voor Plasmafysica, Rijnhuizen, Edisonbaan 14, 3439 MN Nieuwegein, The Netherlands

72. Library, National Institute for Fusion Science, Chikusa-ku, Nagoya 464-01, Japan

73. Library, International Centre for Theoretical Physics, P.O. Box 586, I-34100 Trieste, Italy

74. Library, Centro Richerche Energia Frascati, C.P. 65, I-00044 Frascati (Roma), Italy

75. Library, Plasma Physics Laboratory, Kyoto University, Gokasho, Uji, Kyoto 611, Japan

76. Plasma Research Laboratory, Australian National University, P.O. Box 4, Canberra, A.C.T. 2601, Australia

77. Library, Japan Atomic Energy Research Institute, Naka Fusion Research Establishment, 801-1 Mukoyama, Naka-machi, Naka-gun, Ibaraki-ken, Japan

78. G. A. Eliseev, I. V. Kurchatov Institute of Atomic Energy, P.O. Box 3402, 123182 Moscow, Russia

79. V. A. Glukhikh, Scientific-Research Institute of Electro-Physical Apparatus, 188631 St. Petersburg, Russia

80. I. Shpigel, Institute of General Physics, U.S.S.R. Academy of Sciences, Ulitsa Vavilova 38, Moscow, Russia

81. D. D. Ryutov, Institute of Nuclear Physics, Siberian Academy of Sciences, Sovetskaya St. 5, 630090 Novosibirsk, Russia

82. O. Pavlichenko, Kharkov Physical-Technical Institute, Academical St. 1, 310108 Kharkov, Ukraine

83. Deputy Director, Southwestern Institute of Physics, P.O. Box 15, Leshan, Sichuan, China (PRC)

84. Director, The Institute of Plasma Physics, P.O. Box 1126, Hefei, Anhui, China (PRC)

85. R. H. McKnight, Experimental Plasma Research Branch, Division of Development and Technology, Office of Fusion Energy, Office of Energy Research, ER-542 Germantown, U.S. Department of Energy, Washington, DC 20545

86. E. Oktay, Division of Confinement Systems, Office of Fusion Energy, Office of Energy Research, ER-55 Germantown, U.S. Department of Energy, Washington, DC 20545 
87. W. Sadowski, Fusion Theory and Computer Services Branch, Division of Applied Plasma Physics, Office of Fusion Energy, Office of Energy Research, ER-541 Germantown, U.S. Department of Energy, Washington, DC 20545

88. R. E. Mickens, Atlanta University, Department of Physics, Atlanta, GA 30314

89. M. N. Rosenbluth, University of California at San Diego, La Jolla, CA 92037

90. D. Schnack, SAIC, 10260 Campus Point Drive, San Diego, CA 92121

91. Duk-In Choi, Department of Physics, Korea Advanced Institute of Science and Technology, P.O. Box 150, Chong Ryang-Ri, Seoul, Korea

92. Library of Physics Department, University of Ioannina, Ioannina, Greece

93. C. De Palo, Library, Associazione EURATOM-ENEA sulla Fusione, CP 65, I-00044 Frascati (Roma), Italy

94. Laboratorio Associado de Plasma, Instituto Nacional de Pesquisas Espaciais, Caixa Postal 515, 122201, Sao Jose dos Campos, SP, Brazil

95. Theory Department Read File, c/o D. W. Ross, University of Texas, Institute for Fusion Studies, Austin, TX 78712

96. Theory Department Read File, c/o R. Parker, Director, Plasma Fusion Center, NW 16-202, Massachusetts Institute of Technology, Cambridge, MA 02139

97. Theory Department Read File, c/o R. White, Plasma Physics Laboratory, Princeton University, P.O. Box 451, Princeton, NJ 08543

98. Theory Department Read File, c/o L. Kovrizhnykh, Lebedev Institute of Physics, Academy of Sciences, 53 Leninsky Prospect, 117924 Moscow, Russia

99. Theory Department Read File, c/o B. B. Kadomtsev, I. V. Kurchatov Institute of Atomic Energy, P.O. Box 3402, 123182 Moscow, Russia

100. Theory Department Read File, c/o T. Kamimura, National Institute for Fusion Studies, Nagoya 464, Japan

101. Theory Department Read File, c/o E. Maschke, Departemente de Recherches sur la Fusion Controlée, CEN/Cadarache, F-13108 Saint-Paul-lez Durance, France

102. Theory Department Read File, c/o D. Düchs, JET Joint Undertaking, Abingdon, Oxfordshire OX14 3EA, United Kingdom

103. Theory Department Read File, c/o R. Briscoe, Culham Laboratory, Abingdon, Oxfordshire OX14 3DB, United Kingdom

104. Theory Department Read File, c/o D. Biskamp, Max-Planck-Institut fur Plasmaphysik, Boltzmannstrasse 2, D-8046 Garching, Federal Republic of Germany

105. Theory Department Read File, c/o T. Takeda, Japan Atomic Energy Research Institute, Tokai Fusion Research Establishment, Tokai-mura, Naka-gun, Ibaraki-ken, Japan

106. Theory Department Read File, c/o J. Greene, General Atomics, P.O. Box 85608, San Diego, CA 92186

107. Theory Department Read File, c/o R. Cohen, Lawrence Livermore National Laboratory, P.O. Box 5511, Livermore, CA 94550

108. Theory Department Read File, c/o R. Gerwin, CTR Division, Los Alamos National Laboratory, P.O. Box 1663, Los Alamos, NM 87545

109. R. Aamodt, Lodestar Research Corporation, 2400 Central Avenue, Boulder, CO 80301

110. K. Appert, Centre de Recherches en Physique des Plasmas, Ecole Polytechnique Fédérale de Lausanne, 21 Avenue des Bains, $\mathrm{CH}-1007$ Lausanne, Switzerland

111. M. Ballico, Max-Planck Institut für Plasmaphysik, Boltzmannstrasse 2, D-8046 Garching, Federal Republic of Germany

112. E. Barbato, Associazione EURATOM-ENEA sulla Fusione, Centro Richerche Energia Frascati, C.P. 65, I-00044 Frascati (Roma), Italy

113. A. Bécoulet, Association EURATOM-CEA sur la Fusion Controlée, Centre d'Etudes Nucléaires de Cadarache, B. P. No. 1, F-13108 Saint Paul lez Durances, France

114. S. Bernabei, Plasma Physics Laboratory, Princeton University, P.O. Box 451, Princeton, NJ 08543 
115. A. Bers, Plasma Fusion Center, Massachusetts Institute of Technology, 38-260, Cambridge, MA 02139

116. V. Bhatnagar, JET Joint Undertaking, Abingdon, Oxfordshire OX14 3EA, England

117. P. T. Bonoli, Plasma Fusion Center, Massachusetts Institute of Technology, 175 Albany St., NW17-115, Cambridge, MA 02139

118. M. Brambilla, Max-Planck Institut für Plasmaphysik, Boltzmannstrasse 2, D-8046 Garching, Federal Republic of Germany

119. Dr. Vincent Chan, General Atomics, P. O. Box 85608, San Diego, CA 92186

120. D. Ehst, Building 205, Argonne National Laboratory, 9700 S. Cass Avenue, Argonne, IL 60538

121. D. Faulconer, Laboratoire de Physique des Plasmas, EURATOM-Etat Belge, Ecole Royale Militaire, Avenue de la Renaissance 30, 1040 Brussels, Belgium

122. D. J. Gambier, JET Joint Undertaking, Abingdon, Oxfordshire OX14 3EA, England

123. S. N. Golovato, Plasma Fusion Center, Massachusetts Institute of Technology, 175 Albany Street, NW17-113, Cambridge, MA 02139

124. W. Ho, SAIC, 10260 Campus Point Drive, San Diego, CA 92121

125. J. Hosea, Plasma Physics Laboratory, Princeton University, P.O. Box 451, Princeton, NJ 08543

126. Kwok Ko, Stanford Linear Accelerator Center, P.O. Box 4349, Stanford, CA 94309

127. C. C. Petty, General Atomics, P.O. Box 85608, San Diego, CA 92186

128. C. K. Phillips, Plasma Physics Laboratory, Princeton University, P.O. Box 451, Princeton, NJ 08543

129. M. Porkolab, Plasma Fusion Center, Massachusetts Institute of Technology, 175 Albany Street, NW17-119, Cambrid.ge, MA 02139

130. R. Prater, General Atomics, P.O. Box 85608, San Diego, CA 92186

131. J. Scharer, Electrical and Computer Engineering Department, 1500 Johnson Drive, University of Wisconsin, Madison, WI 53706

132. P. E. Vandenplas, Laboratoire de Physique des Plasmas, EURATOM-Etat Belge, Ecole Royale Militaire, Avenue de la Renaissance 30, 1040 Brussels, Belgium

133. H. Weitzner, Courant Institute for Mathematical Sciences, New York University, 251 Mercer Street, New York, NY 10001

134. J. R. Wilson, Plasma Physics Laboratory, Princeton University, P.O. Box 451, Princeton, NJ 08543

135-182. Given distribution according to OSTI-4500, Magnetic Fusion Energy (Category Distribution UC-427, Theoretical Plasma Physics) 